\title{
PROPRIÉTÉS MAGNÉTIQUES DE L'ÉTAT MÉTALLIQUE ET ÉNERGIE D'INTERACTION ENTRE ATOMES MAGNÉTIQUES
}

\author{
Par M. Louis NÉEL
}

SOMMAIRE. - Une première partie du travail ( $\S_{\text {I à }}$ 10) est consacrée à l'interprétation des expériences de M. Manders sur les variations, en fonction de la température, de la susceptibilité magnétique de quelques solutions solides à base de nickel (Ni et $\mathrm{Al}$ ou Ti, Sn, Sb, V, Mo, W, Cr). On étudie et on interprète les variations, en fonction du titre, de la constante de Curie et du coefficient de paramagnétisme constant superposé. On en déduit que les électrons magnétiques du nickel restent en nombre constant lorsqu'on passe de l'état ferro à l'état paramagnétique.

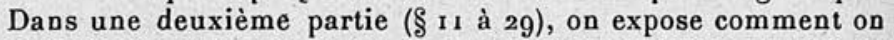
peut définir et calculer une énergie d'interaction magnétique entre deux atomes voisins porteurs de moment, à partir des données expérimentales, soit pour les ferromagnétiques, soit pour les corps à champ moléculaire négatif ( $\mathrm{Pd}$ et $\mathrm{Pt})$, soit pour les corps paramagnétiques à susceptibilité indépendante de la température ( $\mathrm{Mn}, \mathrm{Cr}, \mathrm{Ti}, \mathrm{Mo}, \mathrm{Ru}, \mathrm{Rh}$, etc.). On étudie ensuite les variations de l'énergie d'interaction avec la distance entre les couches magnétiques des atomes interagissant et on montre qu'en première approximation l'énergie d'interaction ne dépend que de cette distance et varie très régulièrement avec elle. Cette conception permet d'interpréter et de relier entre eux un certain nombre de faits expérimentaux dont quelques-uns sont passés en revue.

Enfin, en supposant qu'il existe un couplage entre le réseau cristallin et les spin responsables du magnétisme des métaux, apparaissent des propriétés curieuses qui semblent être un point de départ pour expliquer les propriétés magnétiques compliquées du platine $(\S 18$, i9 et 30$)$. 


\section{INTRODUCTION}

Cet article a pour objet une étude des propriétés magnétiques de l'état métallique, métaux purs et alliages, fondéesur les résultats expérimentaux, en prenant comme base de discussion l'existence du moment atomique et du champ moléculaire. J'étudierai donc, à partir du paragraphe ro, les méthodes de détermination du champ moléculaire c'est-à-dire de l'énergie d'interaction $w$ entre deux atomes magnétiques, et les variations de cette énergie en fonction de la distance des atomes et de la dimension des couches magnétiques. Suivant la grandeur et le signe de $w$, on obtient toute une gamme de propriétés différentes.

Quant au moment atomique, il n'est probablement pas absolument constant, surtout en solution solide, mais en vue d'une étude ultérieure de ses variations, j’ai estimé utile de le supposer invariable, en première approximation, et de reporter d'abord mon attention sur le champ moléculaire seul.

D'ailleurs, l'étude des alliages de nickel apporte des éclaircissements sur une question controversée : celle de la permanence du moment lorsque le nickel passe, au point de Curie, de l'état ferromagnétique à l'état paramagnétique. J'ai utilisé dans cette discussion, pour l'état ferromagnétique les expériences de Sadron (I) et pour l'état paramagnétique les expériences de Manders (2). Ce dernier a fait récemment une étude très soignée et très étendue d'un grand nombre de solutions solides à base de nickel.

Je n'ai essayé d'interpréter que les propriétés des alliages dans lesquels l'atome du métal allié au nickel possède de i à 6 électrons, dans les couches électroniques incomplètes. De plus, j'ai laissé de côté certaines séries d'alliages, par exemple les $\mathrm{Ni}-\mathrm{Pb}$, dans lesquels les limites de solution Ann. de Phys., 1 10 série, t. 5 (Février 1936). 
solide sont si rapprochées qu'il est impossible d'étudier avec certitude, en fonction du titre, les variations des propriétés magnétiques.

§ 1. Interprétation des expériences de Manders. - La représentation habituelle $\left(\frac{1}{\chi}, T\right)$ appliquée aux résultats de Manders montre que les alliages n'obêissent pas à la loi de Weiss, car les graphiques donnent des courbes. Ces courbes ressemblent beaucoup à celles que Mlle Serres a obtenues dans l'étude des ferrites (3), qui s'interprètent par la superposition d'un paramagnétisme variable et d'un paramagnétisme indépendant de la températnre dû à $\mathrm{Fe}^{2} \mathrm{O}^{3}$. Par analogie, j'ai cherché à représenter les expériences sur les alliages de nickel par une formule du type : $\chi=a+\frac{\mathrm{C}}{\mathrm{T}-\theta}$.

$\S 2$. Calcul de $a, \mathrm{C}$ et $\theta$. - La détermination de $a, \mathrm{C}$ et $\theta$ d'après les données expérimentales est d'autant plus difficile qu'il faut exclure la région voisine du point de Curie qui est troublée par les fluctuations du champ moléculaire. La région utilisable commence à $100^{\circ}$ ou $150^{\circ}$ au-dessus du point de Curie : la limite dépend de la concentration et son choix est délicat. L'existence de cette limite ne permet pas d'adopter un procédé automatique de calcul tel que la méthode des moindres carrés. Il faut au contraire avoir sous les yeux l'ensemble des points relatifs à un alliage, reportés dans une représentation graphique sensible. Après différents essais, ¡'ai choisi la méthode suivante : on détermine des valeurs approchées $a^{\prime}, \theta^{\prime}$, des constantes $a, \theta$, par un procédé quelconque, et on reporte sur un graphique les valeurs de: $y=\left(\chi-a^{\prime}\right)\left(\mathrm{T}-\theta^{\prime}\right)$ en fonction de $x=\mathrm{T}-\theta^{\prime}$.

Posons : $a=a^{\prime}+\Delta a^{\prime}, \quad \theta=\theta^{\prime}+\Delta \theta^{\prime}$;

$\Delta a^{\prime}$ et $\Delta \theta^{\prime}$ par hypothèse sont petits de sorte que:

$$
\left(\chi-a^{\prime}\right)\left(\mathrm{T}-\theta^{\prime}\right)=\mathrm{C}+(\mathrm{T}-\theta) \Delta a^{\prime}+\frac{\mathrm{C}}{\mathrm{T}-\theta} \Delta \theta^{\prime},
$$


car $\mathrm{T}-\theta^{\prime}$ se confond sensiblement avec $\mathrm{T}-\theta$; on obtient ainsi l'hyperbole :

$$
y=\mathrm{C}+x \Delta a^{\prime}+\frac{\mathrm{C}}{x} \Delta \theta^{\prime}
$$

Si les valeurs provisoires adoptées sont correctes, l'hyperbole se réduit à la droite : $y=\mathrm{C}$. Si $\theta^{\prime}$ seul est exact, on obtient une droite dont la pente donne $\Delta a^{\prime}$. Si $a^{\prime}$ seul est exact, on obtient une hyperbole équilatère dont l'une des

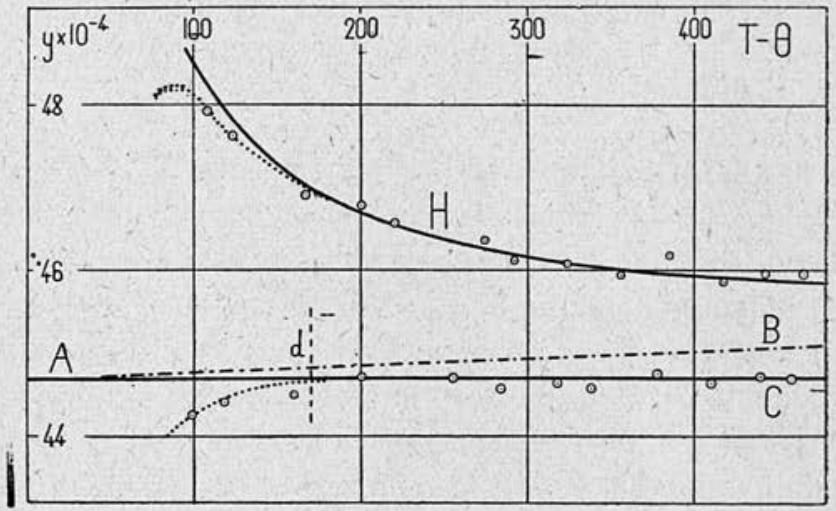

Fig. 1 .

asymptotes est la droite $y=\mathrm{C}$. Les anomalies de la région du point de Curie se traduisent par une chute rapide de la courbe au voisinage de $\mathrm{T}-\theta=100^{\circ}$. Avec un peu d'habitude, l'hyperbole représentative de la courbe $y$ et ses asymptotes se tracent facilement $\left({ }^{1}\right)$ d'où $\mathrm{G}$ et $\Delta a^{\prime}$. La précision avec laquelle se fait cette opération s'apprécie facilement.

A titre d'exemple, je donne le graphique (fig. I) correspondant à un alliage $\mathrm{Ni}-\mathrm{Al}$, à 2,2 1 o/o d'aluminium. La position

(1) On peut utiliser la propriété suivante : en coordonnées obliques avec les asymptotes pour axes, la tangente au point $\left(x_{0}, y\right)$ coupe les axes aux points $\left(0,2 y_{0}\right)$ et $\left(2 x_{0}, 0\right)$. 
des points a d'abord été calculée avec les valeurs provisoires : $\theta=245^{\circ}$ et $a=0,8 \times 10^{-6}$. L'hyperbole $\mathrm{H}$ les représente convenablement. L'une des asymptotes est la droite $\mathrm{AB}$, d'où en A, la constante de Curie définitive : $44,7 \cdot 10^{-4}$. La pente de l'asymptote donne $\Delta a^{\prime}=0,1 \cdot 10^{-6}$, et un point quelconque de l'hyperbole $\Delta \theta=8^{\circ}$. Comme vérification, j'ai repris les calculs avec les valeurs corrigées : $\theta=253^{\circ}$ et $a=0,9 \cdot 10^{-6}$. Les points reportés s'alignent bien sur la droite horizontale $\Lambda$ C, d'ordonnée $y=44,7 \times 10^{-4}$. Sur la figure $\mathrm{r}$, les anomalies du voisinage du point de Curie sont encore sensibles pour $\mathrm{T}-\theta=150^{\circ}$ : les points représentatifs sont au-dessous de la droite AC (portion Ad du graphique).

§ 3. Application aux expériences de Manders. - Les résultats obtenus sont résumés dans le tableau I dont les différentes colonnes indiquent successivement : le titre spécifique, le titre atomique, le point de Curie $\theta$, la constante de Curie C et le coefficient de paramagnétisme constant $a$, rapportés à I g. d'alliage. Les deux dernières colonnes donnent les valeurs de $\mathrm{C}$ et de $a$, rapportées à la quantité d'alliage contenant un nombre d'atomes égal au nombre d'Avogadro.

J'ai complété ce tableau par quelques-uns des résultats d'Alder (4) sur les alliages de nickel et de cuivre et par quelques chiffres provenant du travail de Safranek (5) sur les Ni-Gr. M. Weiss avait depuis longtemps remarqué que les expériences de Safranek indiquaient la superposition d'un paramagnétisme constant dont il avait calculé la valeur. Ce sont ces valeurs, obligeamment communiquées par $\mathrm{M}$. Weiss, qui figurent dans le tableau I.

La discussion des données expérimentales qui suit implique que la formule :

$$
\chi=a+\frac{\mathrm{C}}{\mathrm{T}-\theta},
$$

n'est pas simplement empirique, mais traduit un fait fondamental : la superposition de deux phénomènes essentielle- 
PROPRIÉtÉS MaGNÉTIQUES DE L'ÉTAT MÉTALLiQUE

TABLEAU I

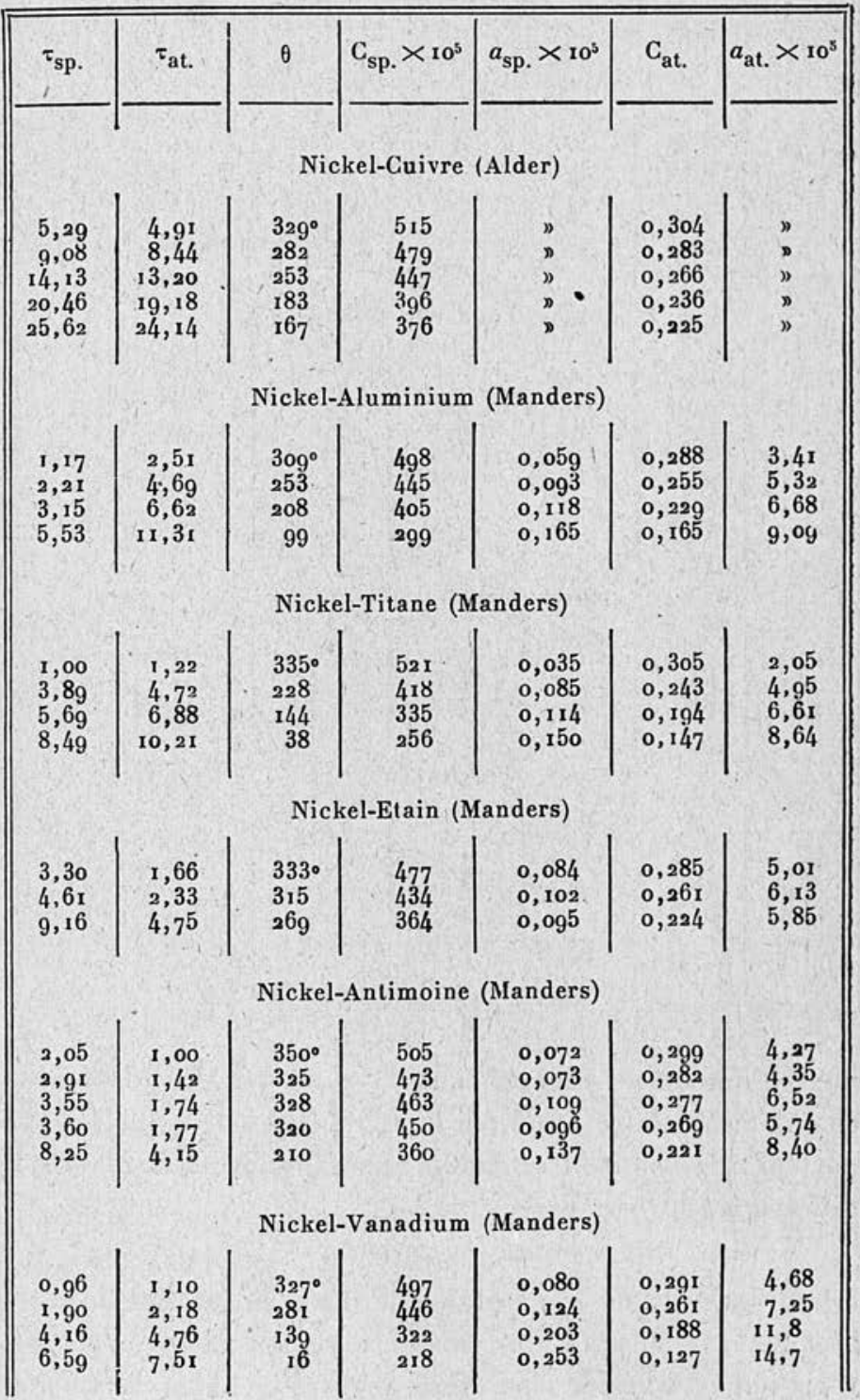




\begin{tabular}{|c|c|c|c|c|c|c|}
\hline$\tau_{\text {sp. }}$ & $\tau_{\text {at. }}$ & $\theta$ & $\mathrm{C}_{\text {sp. }} \times 10^{5}$ & $a_{\text {sp. }} \times 10^{5}$ & $\mathrm{C}_{\text {at. }}$ & $a_{\text {at. }} \times 10^{5}$ \\
\hline \multicolumn{7}{|c|}{ Nickel-Molybdène (Manders) } \\
\hline 0,78 & 0,48 & $365^{\circ}$ & 535 & 0,040 & 0,315 & 2,35 \\
\hline 0,92 & 0,57 & 352 & 517 & 0,047 & $0,3 \circ 5$ & 2,77 \\
\hline 2,45 & $1,5 i$ & 318 & 477 & $0,07^{2}$ & 0,282 & 4,26 \\
\hline 3,25 & 2,01 & 281 & 423 & 0,102 & 0,251 & 6,06 \\
\hline 8,05 & 5,08 & 226 & 208 & 0,295 & 0,126 & 17,9 \\
\hline \multicolumn{7}{|c|}{ Nickel-Tungstène (Manders) } \\
\hline 1,43 & 0,46 & $355^{\circ}$ & 520 & 0,055 & 0,308 & 3,26 \\
\hline 2,77 & 0,90 & 335 & 494 & 0,065 & 0,295 & 3,89 \\
\hline 2,77 & 0,90 & 335 & $49^{2}$ & 0,070 & 0,294 & 4,19 \\
\hline 3,00 & $0,9^{8}$ & 332 & 489 & 0,078 & 0,293 & 4,68 \\
\hline 6,00 & 2,00 & $29 \mathrm{I}$ & $43 i$ & 0,107 & 0,264 & 6,55 \\
\hline 10,00 & 3,42 & 217 & 347 & 0,148 & 0,219 & 9,32 \\
\hline 20,00 & 7,38 & 6 & 133 & 0,222 & 0,090 & 15,1 \\
\hline \multicolumn{7}{|c|}{ Nickel-Chrome (Manders) } \\
\hline $\mathrm{r}, 02$ & $\mathrm{I}, \mathrm{I} 5$ & $340^{\circ}$ & 520 & 0,088 & 0,305 & 5,16 \\
\hline 2,92 & 3,29 & 263 & 466 & 0,163 & $0,27^{3}$ & 9,53 \\
\hline 4,50 & 5,05 & 217 & 427 & $0,22 I$ & 0,249 & 12,9 \\
\hline 1,51 & $\mathbf{1}, 70$ & $331^{\circ}$ & 500 & 0,100 & 0,293 & 5,86 \\
\hline $2,9^{2}$ & 3,29 & 269 & 428 & 0,190 & 0,250 & II, I \\
\hline 4,06 & 4,57 & 229 & 422 & 0,200 & 0,246 & II, 7 \\
\hline 6,02 & $6,7^{5}$ & 117 & 364 & 0,270 & 0,212 & 15,7 \\
\hline 8,00 & 8,94 & 94 & 307 & 0,3 io & 0,178 & 18,0 \\
\hline 10,05 & II, 2 I & -10 & 247 & 0,365 & 0,143 & 21,1 \\
\hline
\end{tabular}

ment différents et indépendants : $a$ ) un paramagnétisme à champ moléculaire; $b$ ) un paramagnétisme constant. Certains porteurs de moment contribuent au premier et d'autres, différents, provoquent le second.

\$4. Variation de la constante de Curie en fonction du titre atomique. - Jusqu'à des titres atomiques de ro o/o cette variation est linéaire, comme le montre la figure 2 , où ont 
été reportés quelques-uns des résultats du tableau précédent. J'ai calculé de la manière suivante la pente de ces droites, c'est-à-dire l'abaissement $\Delta \mathrm{C}$ de la constante de Curie, pour une variation de titre égale à $\mathrm{I}$ (voir fig. 3 ). Soit $\mathrm{C}=0,3_{2} 3$

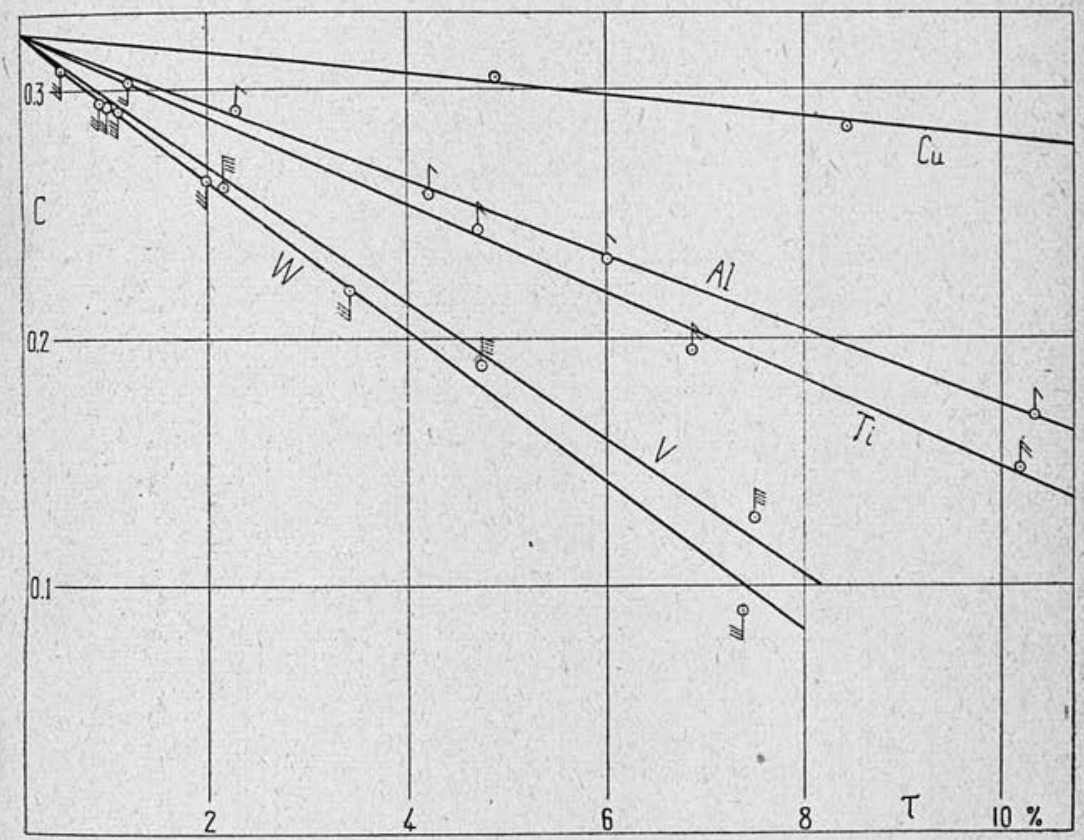

Fig. 2. - Variation de la constante de Curie en fonction de la concentration, pour quelques séries d'alliages.

la constante de Curie du nickel pur, soit $\mathrm{C}-\delta \mathrm{C}$ la constante de Curie d'un alliage de titre $\tau$, d'où une pente $\frac{\delta G}{\tau}$. J'ai pris la moyenne de ces valeurs, en attribuant à chacune un poids proportionnel au titre, d'où :

$$
\Delta \mathrm{C}=\frac{\Sigma \tau \frac{\delta \mathrm{G}}{\tau}}{\Sigma \tau}=\frac{\Sigma \delta \mathrm{G}}{\Sigma \tau}
$$




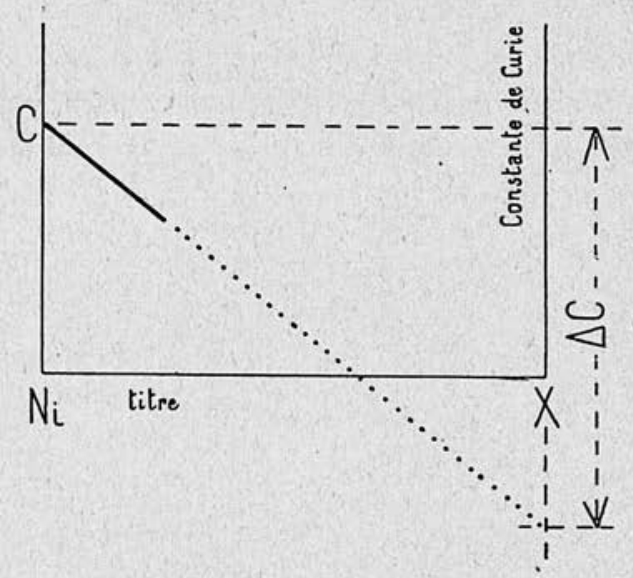

Fig. 3. - Détermination de $\Delta \mathrm{G}$.

Les valeurs ainsi calculées sont réunies dans le tableau 2 :

TABleau 2

\begin{tabular}{|c|l|}
\hline Métal & $-\Delta \mathrm{C}$ \\
\hline Cu & 0,429 \\
$\mathrm{Al}$ & $1,4 \mathrm{I}$ \\
$\mathrm{Ti}$ & 1,75 \\
$\mathrm{Sn}$ & 2,29 \\
V & 2,73 \\
Sb & 2,65 \\
Mo & 3,04 \\
W & 3,09 \\
$\mathrm{Cr}$ & 1,46 \\
\hline
\end{tabular}

§ 5. Expériences de Sadron. Leur interprétation. - Un rapprochement s'impose entre les résultats du paragraphe précédent et l'étude de l'aimantation à saturation des mêmes alliages faite par Sadron (I). Cet auteur a établi que l'aimantation à saturation variait linéairement avec le titre. En supposant que cette variation linéaire se conservait dans tout 
PROPRIÉtÉS MAGNÉTIQUES DE L'ÉTAT MÉTALLIQUe 241

Tableau 3

\begin{tabular}{|c|c|}
\hline \hline Métal & $\Delta \mu_{w}$ \\
\cline { 1 - 2 } $\mathrm{Cu}$ & 5,09 \\
$\mathrm{Zn}$ & $\mathrm{10,3}$ \\
$\mathrm{Al}$ & 15,2 \\
$\mathrm{Sn}$ & 21,0 \\
$\mathrm{~V}$ & 26,0 \\
Mo & 30,0 \\
\hline
\end{tabular}

l'intervalle des concentrations, il a calculé l'abaissement $\Delta \mu_{w}$ de l'aimantation à saturation pour une variation de titre atomique égale à $\mathrm{I}$. J'ai rassemblé dans le tableau 3 pour différents métaux, ces abaissements $\Delta \mu_{w}$ exprimés en magnétons de Weiss.

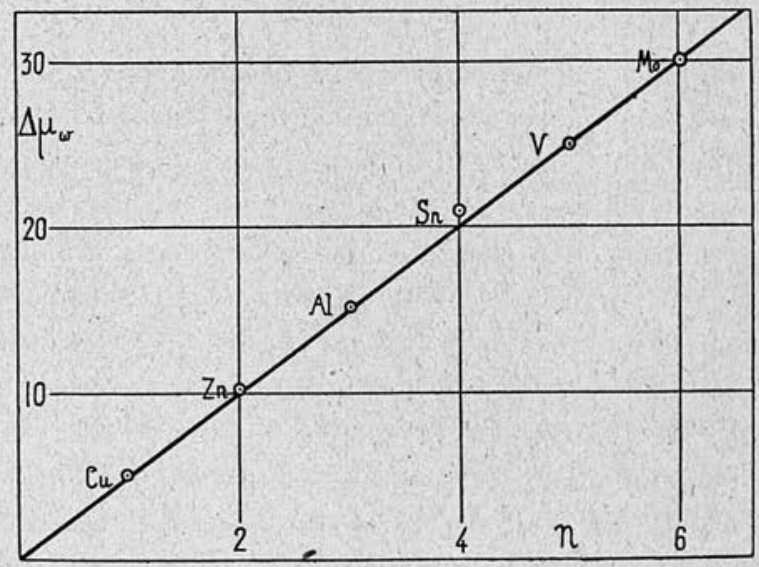

Fig. 4. - Abaissement atomique du moment dans les alliages.

Toutes ces valeurs ont été empruntées au mémoire de Sadron, sauf celle du molybdène que je dois à l'obligeance de MM. Forrer et Marian (expériences encore inédites). 
Sadron a démontré que $\Delta \mu_{w}$ ne dépendait que de la configuration électronique extérieure du métal ajouté au nickel. De plus, la figure 4 montre que $\Delta \mu_{w}$, en ordonnées, varie proportionnellement aux nombres des électrons de la couche incomplète qui a été porté en abscisses.

Ces résultats expérimentaux peuvent ainsi se résumer : tout se passe comme si, en ajoutant un métal étranger au nickel :

a) Les atomes du métal étranger remplaçaient des atomes de nickel dépourvus de moment $\left(^{1}\right)$;

b) Les électrons du métal étranger se disposaient antiparallèlement à ceux du nickel.

Le mécanisme du phénomène nous échappe. Les hypothèses proposées ci-dessus sont uniquement des hypothèses de travail. La théorie de l'état métallique n'est pas, jusqu'ici, assez développée pour être de quelque utilité; il faut bien se résigner à cette manière de procéder.

§. Signification des expériences de Manders. - J'appliquerai les hypothèses de travail proposées ci-dessus. De même que pour l'aimantation à saturation, je représente les abaissements atomiques $\Delta \mathrm{C}$ de la constante de Curie en fonction du nombre des électrons des couches incomplètes du métal ajouté au nickel. $\Delta \mathrm{C}$ varie, comme $\Delta \mu_{w}$, proportionnellement au nombre des électrons (figure 5). Le vanadium et l'antimoine possèdent le mêmè nombre d'électrons extérieurs : ils fournissent la même valeur de $\Delta \mathrm{C}$; même remarque pour le molybdène et le tungstène. La régularité des résultats est moins grande que pour les moments à basse température car la détermination des constantes de Curie est moins précise que celle des moments.

En particulier, on remarque que l'abaissement dû au

(1) Cette hypothèse a été primitivement énoncée par Dorfman (6). 
cuivre $(0,429)$ est relativement fort éloigné de la valeur qui correspond à la droite tracée $(0,5,3)$. Or, les valeurs d'Alder ont été obtenues sans correction de paramagnétisme constant superposé. L'étude des résultats d'Alder montre qu'ils sont compatibles avec l'hypothèse d'un petit paramagnétisme constant superposé; les constantes de Curie sont

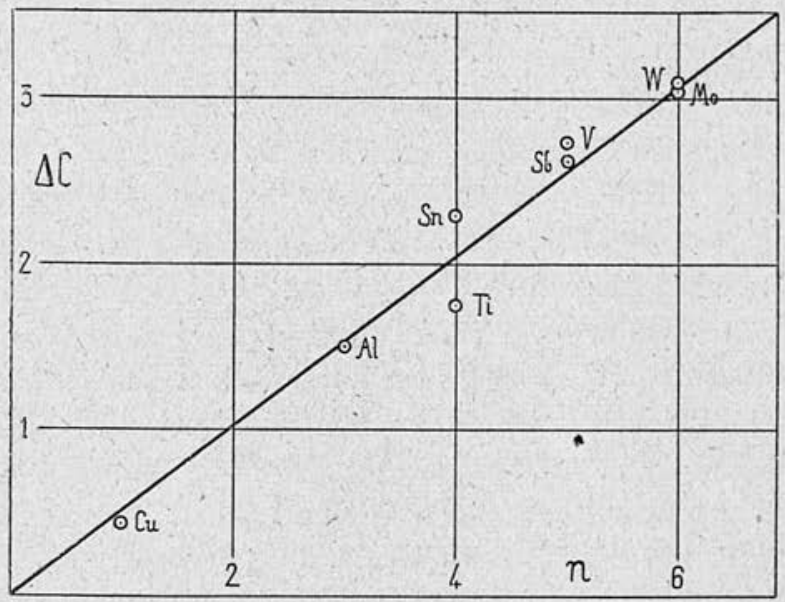

Fig. 5. - Abaissement atomique de la constante de Curie dans les alliages.

alors modifiées et l'abaissement atomique de la constapte de Curie devient voisin de 0,513 .

D'autre part, l'abaissement atomique du chrome $(1,46)$ n'a pas été reporté sur le graphique : il présente avec la droite un écart considérable que j'interpréterai dans le $\$ 27$.

De la droite tracée sur la figure 5 , se déduit un abaissement atomique de la constante de Curie égal à o,5r3 par électron. L'adjonction d'atomes étrangers au nickel annule autant d'électrons magnétiques du nickel que l'on a ajouté d'électrons appartenant à des couches incomplètes. Comme un électron par atome produit une diminution de constante de Curie égale à o, $5_{1} 3$, c'est dire que l'électron par atome 
dont on a annulé la contribution magnétique possédait une constante de Curie égale à 0,513 .

Comme la constante de Curie atomique du nickel pur est égale à 0,323 , j'en déduis que le nombre d'électrons qui contribuent aux propriétés paramagnétiques du nickel est égal à $\frac{0,323}{0,513}=0,63$ électron par atome.

A basse température, l'aimantation à saturation correspond à o,6r magnéton de Bohr, soit un nombre égal d'électrons par atome. Ainsi, dans l'état ferromagnétique, au-dessous du point de Curie, le nombre des électrons effectifs est, à la précision des mesures, égal au nombre des électrons effectifs de l'état paramagnétique, au-dessus du point de Curie. Le nombre des électrons non compensés du nickel est le même, à haute et à basse température.

L'introduction d'un métal étranger dans le nickel fait disparaître progressivement les propriétés magnétiques. A égalité de titre, on ob̂serve les mêmes abaissements relatifs du moment à saturation et de la constante de Curie, d'où identité complète entre les porteurs de moment responsables du ferromagnétisme et ceux qui produisent le paramagnétisme.

Ce résultat est important, car il permet d'éliminer les interprétations des propriétés du nickel qui font appel à une augmentation du nombre des porteurs de l'état ferromagnétique à l'état paramagnétique. C'est ainsi qu'on ne saurait considérer le nickel comme constitué de porteurs à I magnéton de Bohr, car il faudrait, par atome, o, 6 de ces porteurs à basse température et au contraire 0,9 à haute température.

- § 7. Hypothèse de Dorfman (6). - De même, Dorfman envisage le nickel comme un mélange d'atomes neutres possédant un moment nul et d'atomes ionisés avec un moment magnétique de I magnéton de Bohr : soit $40 \%$ o de $\mathrm{Ni}$ et 60 o/o de $\mathrm{Ni}^{+}$, c'est-à-dire à basse température un moment 
moyen de $0,6 \mu_{\mathrm{B}}$. La constante de Curie correspondante est 0,225 beaucoup plus faible que la valeur expérimentale 0,323 . Dorfman attribue cette différence à l'intervention du moment des orbites qui ne seraient plus bloquées à haute température. Ce mécanisme est peu vraisemblable car, a priori, l'annulation de la fraction du moment dû au spin n'entraîne pas nécessairement l'annulation des moments orbitaux; les deux mécanismes sont indépendants $\left.{ }^{(}\right)$.

\section{$\S 8$. Hypothèse de Wolf et de Stoner $(7,8)$. - Suivant} Wolf et Stoner, il faut attribuer deux magnétons de Bohr à ceux des atomes de nickel possédant un moment; $30 \mathrm{o} / 0$ des atomes sont, par exemple, dans l'état ${ }^{3} \mathrm{~F}\left(d^{8} s^{2}\right)$ avec $2 \mu_{-\mathrm{B}}$, les autres ayant un moment nul. La constante de Curie calculée est 0,300 , peu éloignée de la valeur expérimentale 0,323 . Le problèmehth'est ainsi résolu que d'une manière formelle, car il s'agit de savoir pourquoi tous les atomes de nickel ne sont pas identiques. Wolf suggère un équilibre thermique entre l'état ${ }^{3} \mathrm{~F}$ et l'état ${ }^{1} \mathrm{D}$, mais la différence d'énergie entre ces deux états est trop grande pour qu'il existe à la températnre ordinaire une quantité appréciable d'atomes dans l'état ${ }^{1} \mathrm{D}$; de plus, l'équilibre dépendrait de la température. Stoner suggère que normalement les électrons du nickel tendent à se compenser deux à deux, mais que sous l'influence du champ moléculaire positif du nickel s'établit un état d'équilibre caractérisé par o,6 électron non compensé par atome. Ce raisonnement conduit à dénier toute signification à l'identité entre les constantes de Curie du nickel, du palladium et du platine, métaux possédant la même configuration électro-

(1) La rédaction de ce mémoire étant achevée, Sucksmith (Helv. Phys. Acta, r935, 8, p. 205) a montré, par l'étude de l'effet gyromagnétique, que les alliages de nickel et de cuivre, possédant un point de Curie aux environs de la température ordinaire, fournissaient une valeur de $g$ égale à 2, dans l'état paramagnétique. Le moment orbital n'intervient donc pas, ce qui confirme notre conclusion. 
nique extérieure. En effet, le palladium et le platine, possédant un champ moléculaire négatif, auraient ainsi un moment nul. Il est impossible d'éliminer arbitrairement des résultats expérimentaux bien établis qui ne sauraient être attribués à des coïncidences.

La question reste ouverte ; tout ce qui est sûr, c'est qư'il existe, de part et d'autre du point de Curie, environ o, 6 électron non compensé par atome ; il est probable que ces électrons sont couplés deux par deux parallèlement.

§ 9. Origine du paramagnétisme constant superposé des alliages de nickel. - Dans la seconde des hypothèses du $\S 5$, j'attribue la disparition de la contribution magnétique de certains électrons du nickel à la formation de couples d'électrons antiparallèles. Si la liaison n'est pas rigide, il y a déformation sous l'action du champ magnétiqué et apparition d'un paramagnétisme indépendant de la température, d'où le terme $a$ introduit pour représenter les faits expérimentaux.

Des données du tableau $\mathrm{r}$, on déduit que le terme $a$ ne croit pas proportionnellement au titre atomique, mais beaucoup moins rapidement. Un tel résultat est inexplicable si deux électrons, appartenant l'un au nickel et l'autre au métal X, s'annulent complètement et si cette paire une fois formée n'interagit plus avec le reste des porteurs magnétiques. On doit avoir, au contraire, une variation proportionnelle au titre, tout au moins aussi longtemps que la constante de Curie varie linéairement avec le titre.

Il faut se représenter les choses autrement : supposons que tous les électrons appartenant aux couches incomplètes des atomes $\mathrm{X}$ soient dirigés parallèlement à une certaine direction; dirigeons en sens inverse un nombre égal d'électrons appartenant au nickel, de manière à former un assemblage de moment total nul. J'obtiens ainsi un réseau dont les électrons constitutifs restent, par hypothèse, sur les atomes 
dont ils sont originaires. Le nombre des atomes de nickel appartenant à ce réseau est proportionnel au nombre des atomes X.

Les énergies d'interaction qui sont à l'origine de cette disposition antiparallèle sont, au signe près, du même ordre de grandeur que celles qui interviennent dans les ferromagnétiques. Seul le mécanisme d'Heisenberg convient pour les interpréter. Ces énergies varient très rapidement avec la distance des atomes, de sorte que les actions entre atomes proches voisins sont seules nơtables. Les électrons d'un atome X ne peuvent s'opposer qu'aux électrons des atomes de nickel faisant partie des douze voisins immédiats. Je suppose que l'énergie d'interaction est indépendante de la concentration.

Le paramagnétisme de déformation du réseau est proportionnel au nombre d'atomes $\mathrm{X}$ et inversement proportionnel au nombre de liaisons par atome. Ce point sera précisé quantitativement dans le $\$ 24$.

Le nombre de ces liaisons antiparallèles se calcule ainsi : soit $\tau$ le titre atomique du métal $\mathrm{X}$; un atome de nickel dont le moment a été annulé possède dans le réseau cristallin douze voisins, dont l'un est obligatoirement un atome $\mathrm{X}$, mais chacune des onze autres positions peut être aussi occupée par un atome $X$, avec une probabilité $\tau$; le nombre de liaisons par atome de nickel est ainsi I + I I $\tau$, et le paramagnétisme constant de l'alliage est proportionnel à :

$$
\frac{\tau}{1+11 \tau}
$$

Pour vérifier la validité de cette formule, j’ai représenté, sur les figures 6 et $7, a(1+1$ I $\tau)$ en fonction de $\tau$. La proportionnalité se vérifie particulièrement bien pour l'aluminium, le titane et le chrome dont les alliages correspondants ont été préparés jusqu'au titre $\tau=0,1$. L'expérience confirme ainsi le mécanisme proposé. 
Un point est à signaler : j'ai supposé que l'assemblage antiparallèle avait, en l'absence de champ magnétique, un moment total nul; pourquoi les atomes de nickel qui n'appartiennent pas à l'assemblage et qui sont au voisinage immédiat d'un atome $X$, ne viennent-ils pas, eux aussi, se

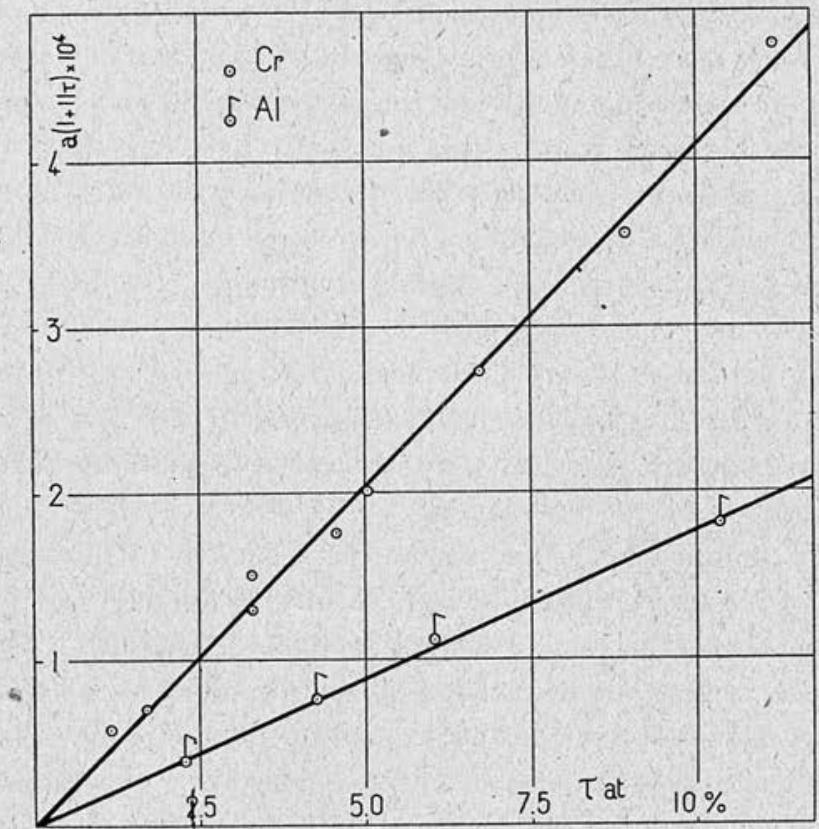

Fig. 6.

placer antiparallèlement à l'atome X ? Précisons par un exemple; soit une solution très étendue d'aluminium dans du nickel ; d'après l'hypothèse faite, chaque atome d'aluminium, pourvu de trois électrons, s'associera antiparallèlement à 3 électrons appartenant à des atomes de nickel. Pourquoi les douze atomes de njickel qui entourent l'atome d'aluminium et qui possèdent $12 \times 0,6=7,2$ électrons non compensés ne se disposent-ils pas tous antiparallèlement à 
l'atome d'aluminium ? Au contraire, d'après les résultats expérimentaux, il existe une sorte de saturation de la liaison antiparallèle dont les propriétés sont ainsi très voisines de celles de la liaison homopolaire. Toutes deux s'interprètent par la mise en commun de deux électrons dans la même cellule d'extension en phase. Ces assemblages de moment total nul se rapprochent beaucoup par leurs propriétés, de

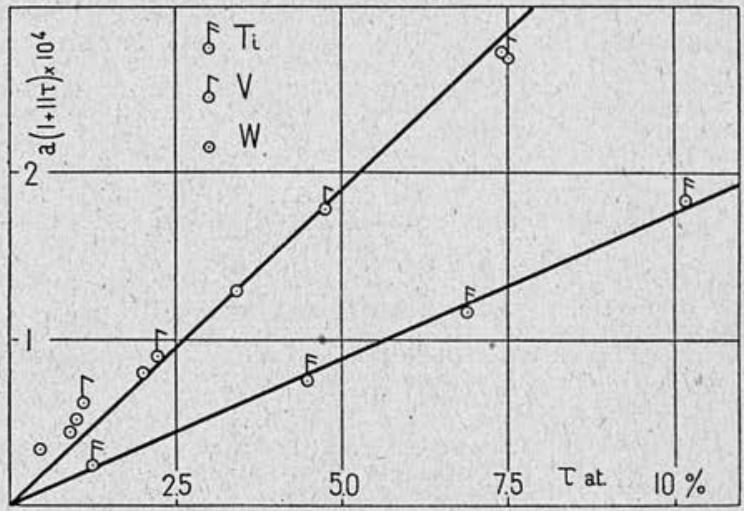

Fig. 7 .

ceux qu'on obtient, dans la théorie du paramagnétisme constant de Pauli, par application de la statistique de FermiDirac.

§ 10. Remarque sur les théories du magnétisme. - On peut attaquer de deux façons différentes les problèmes du magnétisme : soit partir d'un gaz électronique obéissant à la statistique de Fermi-Dirac et introduire des perturbations pour tenir compte de la présence des ions dans le réseau; cette méthode est utilisée dans les problèmes de conductibilité, elle a fourni la clé du paramagnétisme des métaux alcalins. On peut aussi prendre des atomes isolés puis les rapprocher en tenant compte de leurs interáctions : c'est Ann. de Phys., $11^{\mathrm{e}}$ série, t. 5 (Février 1936). 
ainsi que l'on a entrepris avec succès le problème du ferromagnétisme.

J'estime qu'aussi bien pour le paramagnétisme constant que pour le ferromagnétisme et surtout dans l'étude des alliages, il est plus avantageux d'utiliser uniformément la seconde méthode. Elle conduit à dissocier le moment et le champ moléculaire et à supposer implicitement une permanence du moment qui n'est sans doute pas toujours réalisée. En l'absence de données expérimentales suffisantes, je n'étudierai pas les variations possibles du moment; je me bornerai à dégager les données actuellement acquises sur les énergies d'interaction.

§11. Différentes manières de calculer les énergies d'interaction, - J'ai utilisé les procédés suivants :

a) Au moyen de la constante de Curie et du point de Curie pour les corps qui obéissent à la loi du paramagnétisme de Weiss comme : $\mathrm{Ni}$, Co, $\mathrm{Fe}, \mathrm{Pt}, \mathrm{Pd}$.

b) Par une extension de la méthode précédente aux alliages, pour obtenir l'énergie des liaisons mixtes: $\mathrm{Ni}$ - Co, $\mathrm{Ni}-\mathrm{Fe}$ et $\mathrm{Fe}-\mathrm{Co}$.

c) Par la susceptibilité des métaux à paramagnétisme indépendant de la température comme : $\mathrm{Cr}, \mathrm{Mn}, \mathrm{V}, \mathrm{Ti} \ldots$ etc.

d) Par l'étude du paramagnétisme constant superposé des alliages de nickel pour calculer l'énergie d'interaction des liaisons mixtes $: \mathrm{Ni}-\mathrm{V}, \mathrm{Ni}-\mathrm{Ti}$.

\$12. Bases générales du calcul. - Comme aucune théorie quantique n'étudie l'aimantation d'un groupe de porteurs magnétiques quel que soit le signe des interactions, j'utiliserai un modèle classique que j’ai déjà employé (9) et qui fournit des résultats cohérents. Je prendrai comme porteurs, les atomes, et je supposerai que chaque alome possède $q$ électrons non composés couplés parallèlement d'une façon absolument rigide, de sorte que le moment magnétique 
résultant de l'atome est égal à $q \mu_{\mathrm{B}}{ }^{(1)}$. Dans le cas du nickel, on est amené à-supposer que tous les atomes ne sont pas identiques. J'appliquerai uniformément la statistique de Langevin.

J'appellerai $-w \cos \alpha$ l'énergie d'interaction entre deux électrons non compensés quelconques situés chacun sur deux atomes proches voisins dont les moments magnétiques font entre eux un angle $\alpha$. Tous les électrons non compensés d'un atome donné sont liés d'une façon identique à un électron quelconque appartenant à un atome différent. Donc, entre deux atomes proches voisins, portant chacun $q$ électrons, l'énergie totale d'interaction est : $-q^{2} w \cos \alpha$. $w$ peut être positif ou négatif; il est nul pour deux atomes qui ne sont pas proches voisins.

J'ai admis les hypothèses précédentes pour être capable, dans tous les cas, de pousser les calculs jusqu'au bout afin de faire une exploration aussi complète que possible du sujet. Evidemment ces calculs demanderaient à être repris par une méthode rigoureuse qui permettrait de serrer les phénomènes de plus près.

§13. Formules valables à haute température.-Dans ce cas, quel que soit le signe de $w$, la substance obéit à la loi de Weiss. Soit un réseau comprenant au total $\mathrm{N}$ atomes de moment $q \mu_{\mathrm{R}}$, possédant chacun $2 p$ voisins, l'aimantation de l'ensemble s'écrit $\left({ }^{2}\right)$ :

$$
J=\frac{1}{3} \frac{N q^{2} \mu_{\mathrm{B}}^{2} \mathrm{H}}{k \mathrm{~T}-\frac{2 p q^{2} w}{3}}
$$

(1) $\mu_{\mathrm{B}}=\mathrm{I}$ magnéton de Bohr $=5564$ C. G. S.

() J'aboutis à la loi de Langevin car je suppose que deux porteurs voisins peuvent occuper relativement des positions quelconques, hypothèse essentielle pour trouver, avec $w$ négatif, un paramagnétisme indépendant de la température, au voisinage du zéro absolu. 
La constante de Curie et le point de Curie s'écrivent :

$$
\mathrm{C}=\frac{\mathrm{I}}{3} \frac{\mathrm{N} q^{2} \mu_{\mathrm{B}}^{2}}{k} \quad \Theta=\frac{2 p q^{2} w}{3 k}
$$

La formule 3 est identique à la formule 94 de ma thèse (9) au facteur $2 p$ près introduit ci-dessus pour tenir compte des $2 p$ voisins. Je l'avais établie dans un cas particulier mais il est très facile de généraliser.

Des formules 4 on déduit :

$$
\frac{w}{N \mu_{B}^{2}}=\frac{1}{2 p} \frac{\theta}{\mathrm{C}}
$$

formule qui permet de calculer $w$ d'après les données expérimentales $\theta$ et $\mathrm{C}$.

Pour appliquer la formule 5, il n'est pas nécessaire que tous les atomes soient identiques. En effet, au lieu de répartir les $\mathrm{N} q$ électrons magnétiques en nombre égal sur chacun des $\mathrm{N}$ atomes, répartissons-les également entre $\omega_{\mathrm{N}}$ seulement $(0<\omega<\mathrm{r})$; de sorte que $(\mathbf{1}-\varpi) \mathrm{N}$ atomes ont un moment nul. Le moment des atomes magnétiques sera $\frac{q \mu_{B}}{\varpi}$ et l'énergie d'interaction entre deux de ces atomes voisins sera $q^{2} \frac{w}{w^{2}} \cos \alpha$. Le nombre des voisins sera réduit à $2 p$ ๘. Les formules 4 seront remplacées par les suivantes :

$$
\mathrm{C}=\frac{1}{3} \frac{N \varpi q^{2} \mu_{\mathrm{B}}^{2}}{k \varpi^{2}} \quad \text { et } \quad \Theta=\frac{2 p \varpi w q^{2}}{3 \varpi^{2} k}
$$

mais la formule 5 est toujours valable car $\dot{C}$ et $\Theta$ sont tous deux divisés par ๘. On peut ainsi l'appliquer avec confiance notamment dans le cas du nickel où il y a de fortes raisons de croire que les atomes ne sont pas identiques.

$\S 14$. Application de la formule 5 aux métaux suivant la loi de Weiss. - Les éléments du calcul et les résultats sont rassemblés dans le tableau 4. Les métaux envisagés cristallisent 
tous dans le système du cubeà faces centrées; le nombre des proches voisins est donc uniformément de douze.

TABLEaU 4

\begin{tabular}{|c|c|c|c|c|c|c|}
\hline Elément & C & & $\theta$ & Auteur & $\frac{w}{N \mu_{B}^{2}}$ & $\times 10^{-2}$ \\
\hline $\begin{array}{l}\mathrm{Ni} \\
\mathrm{Co} \\
\mathrm{Fe} \gamma \\
\text { Fey } \\
\mathrm{Pt} \\
\mathrm{Pd}\end{array}$ & $\begin{array}{l}0,3_{2} 3 \\
1,14 \\
3,3 \\
9,3 \\
0,318 \\
0,321\end{array}$ & 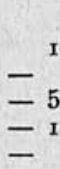 & $\begin{array}{l}650^{\circ} \\
400 \\
730 \\
500 \\
096 \\
228\end{array}$ & $\begin{array}{c}\text { Preuss (I0) } \\
\text { ” } \\
\text { Peschard (II) } \\
\text { Kopp (I2) }\end{array}$ & $\frac{-}{-}$ & $\begin{array}{l}\mathrm{I}, 67 \\
\mathrm{I}, 02 \\
0,18 \\
0,49 \\
2,88 \\
0,59\end{array}$ \\
\hline
\end{tabular}

Les constantes de Curie utilisées dans le tableau 4 sont rapportées à l'atome-gramme, $\mathrm{N}$ est donc égal au nombre d'Avogadro: $6,06 \times 10^{23}$.

J'ai cité deux valeurs relatives au $\mathrm{Fe}_{\gamma}$, leur accord est médiocre. La valeur correspondante de $w$ n'indique donc qu'un ordre de grandeur.

§15. Extension de la théorie aux alliages. - Soit une solution solide entre deux métaux A et B, dont les moments, par atome, sont $\mu_{\mathrm{A}}$ et $\mu_{\mathrm{B}}$; aux énergies d'interaction $w_{\mathrm{AA}}$ et $w_{\mathrm{BB}}$, correspondant respectivement aux actions entre deux atomes A voisins et entre deux atomes B voisins, il faut ajouter une énergie d'interaction $w_{\mathrm{AB}}$ correspondant à l'action entre un atome A et un atome B. Dans le cas d'une répartition statistique des atomes, un atome quelconque est entouré de $2 p \mathrm{P}$ atomes $\mathrm{A}$ et de $2 p Q$ atomes $\mathrm{B}$, en désignant par $\mathrm{P}$ et $Q$ les titres atomiques des atomes $A$ et $B \quad(P+Q=I)$.

Si les atomes de l'alliage n'étaient pas couplés, l'aimantation totale serait la somme des aimantations partielles • $\partial_{A}$ et $\partial_{B}$ des deux constituants telles que :

$$
\partial_{\mathrm{A}}=\frac{\mathrm{C}_{\mathrm{A}} \mathrm{H}}{\mathrm{T}} \text { et } \partial_{\mathrm{B}}=\frac{\mathrm{C}_{\mathrm{B}} \mathrm{H}}{\mathrm{T}} .
$$


Pour tenir compte des actions mutuelles, en négligeant les fluctuations, il faut ajouter au champ magnétique extérieur un champ moléculaire. Le champ moléculaire qui agit sur les atomes $\mathrm{A}$ est de la forme $: a J_{A}+b J_{B}$, et celui qui agit sur les atomes B est de la forme : $b^{\prime} \mathscr{J}_{\mathrm{A}}+c \mathfrak{J}_{\mathrm{B}}$. On trouve :

$$
a=\frac{2 p w_{\mathrm{AA}}}{N \mu_{\mathrm{A}}^{2}} \quad b=b^{\prime}=\frac{2 p w_{\mathrm{AB}}}{\mathrm{N} \mu_{\mathrm{A}} \mu_{\mathrm{B}}} \quad c=\frac{2 p w_{\mathrm{BB}}}{\mathrm{N} \mu_{\mathrm{B}}^{2}}
$$

et l'on a :

$$
\mathscr{J}_{\mathrm{A}}=\frac{\mathrm{C}_{\mathrm{A}}}{\mathrm{T}}\left(\mathrm{H}+a J_{\mathrm{A}}+b \mathfrak{J}_{\mathrm{B}}\right) \text { et } \mathfrak{J}_{\mathrm{B}}=\frac{\mathrm{C}_{\mathrm{B}}}{\mathrm{T}}\left(\mathrm{H}+b J_{\mathrm{A}}+c \mathfrak{J}_{\mathrm{B}}\right)
$$

d'où l'on tire facilement :

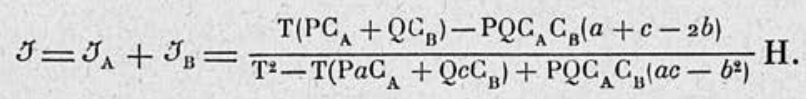

J'ai déjà montré ( 13 ) que cette formule représentait bien l'ensemble des résultats expérimentaux relatifs aux Ni-Co, seul cas où il est légitime de l'appliquer dans tout l'intervalle des concentrations. Elle permet aussi d'interpréter l'allure générale des propriétés magnétiques des Platine-Cobalt (9, p. roo).

Dans la représentation $\left(\frac{\mathrm{I}}{\%}, \mathrm{~T}\right)$, la formule 9 représente une courbe ; expérimentalement on a étudié une région d'une centaine de degrés au-dessus du point de Curie et ce qu'on interprète comme une droite n'est que la tangente à la courbe 9 au point de Curie $\left({ }^{1}\right)$ dont l'équation peut s'écrire sous la forme : $\frac{\mathrm{I}}{\chi}=\frac{\mathrm{T}-\theta^{\prime}}{\mathrm{C}^{\prime}}$.

(1) Sous réserve, naturellement, des anomalies produites par les fluctuations du champ moléculaire qui viennent se superposer aux phénomènes décrits ci-dessus, mais, qui, heureusement, ne s'étendent que dans un domaine de température assez limité (elles disparaissent à $100^{\circ}$ au-dessus du point de Curie). 
\$16. Calcul de $w_{\mathrm{AB}}$ d'après les données expérimentales. Si la concentration du métal B est petite, on a :

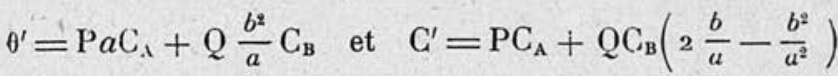

soit, en fonction du titre, une variation linéaire du point de Curie et de la constante de Curie apparente. Prolongeons les droites obtenues jusqu'à $Q=1$; soit $\theta^{n}$ et $C^{\prime \prime}$ les valeurs de $\theta^{\prime}$ et $C^{\prime}$ correspondant à $Q=\mathrm{I}$, d'après (II) on a :

$$
\frac{\mathrm{C}^{\prime \prime}}{\theta^{\prime \prime}}=\frac{2}{b}-\frac{1}{a} \quad \text { ou } \quad b=\frac{2}{\frac{\mathrm{C}^{\prime \prime}}{\theta^{\prime \prime}}+\frac{\mathrm{C}_{\mathrm{A}}}{\theta_{\mathrm{A}}}}
$$

en remarquant que pour le métal A pur, de constante de Curie $\mathrm{C}_{\mathrm{A}}$ et de point de Curie $\theta_{\mathrm{A}}$, on a $: a=\frac{\theta_{\mathrm{A}}}{\mathrm{C}_{\mathrm{A}}}$. $\mathrm{C}^{\prime \prime}$ et $\theta^{\prime \prime}$ se déterminent expérimentalement en extrapolant les tangentes initiales aux courbes de variation de la constante de Curie et du point de Curie en fonction du titre. J'ai appliqué cette méthode pour calculer les énergies d'interaction des liaisons mixtes $w_{A B}: \mathrm{Ni}-\mathrm{Co}, \mathrm{Ni}-\mathrm{Fe}$, Co-Fe, d'après les données expérimentales de Preuss (I0), de Peschard (II) et de Bloch (I4).

Dans le calcul précédent, $w_{\mathrm{AB}}$ représente l'énergie totale d'interaction entre deux moments $\mu_{A}$ et $\mu_{B}$. Pour avoir des valeurs comparables aux $w$ du $\S 14$, il faut exprimer $w_{A B}$ au moyen de l'énergie $w_{\mathrm{AB}}^{\prime}$ d'interaction de deux électrons, portés l'un par l'atome A et l'autre par l'atome B. Posons $\mu_{\mathrm{A}}=q \mu, \mu_{\mathrm{B}}=q^{\prime} \mu$ en désignant par $\mu$ le magnéton de Bohr. On a immédiatement : $w_{\mathrm{AB}}=q q^{\prime} w_{\mathrm{AB}}^{\prime}$. D'où, d'après la formule 8, puisque le facteur $q q^{\prime}$ disparait hait et bas :

$$
b=\frac{2 p w_{\mathrm{AB}}^{\prime}}{\mathrm{N} \mu^{2}} .
$$

Le tableau 5 donne les valeurs de $C^{\prime \prime}, \theta^{\prime \prime}, w_{A B}^{\prime}$ correspondant à différentes liaisons. Le système cristallin étant le cube à faces centrées, on a toujours : $2 p=12$. 
Tableau 5

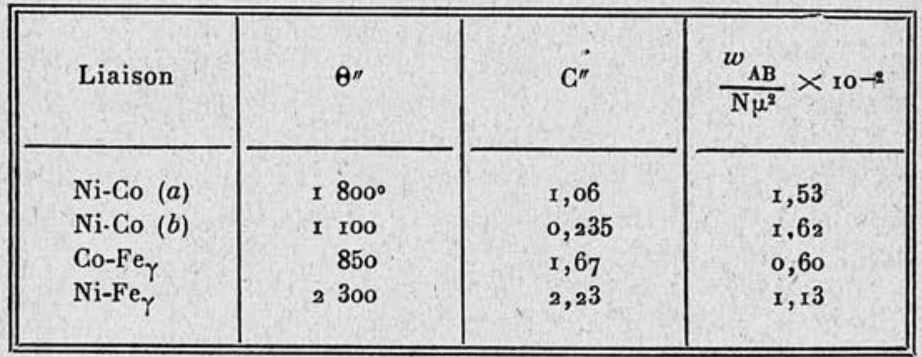

Dans les Ni-Co, on peut tracer les tangentes aux courbes expérimentales, soit en partant du nickel $(a)$, soit en partant du cobalt $(b)$; les deux valeurs correspondantes de $w_{\mathrm{AB}}^{\prime}$ sont concordantes. Pour les solutions solides contenant du fer, je me suis borné à tracer les tangentes en partant soit du cobalt, soit du nickel, car en partant du fer, l'alliage se trouve * dans la phase $\alpha$ ou dans la phase $\gamma$ suivant la température et dans ce dernier cas, surtout, les constantes de Curie observées sont très peu sûres.

§17. Cas des basses températures. - La formule 3 n'est plus valable $\left({ }^{1}\right)$ lorsque $T$ est plus petit que la valeur absolue de $\theta$. Quand $w$ est positif, la substance est ferromagnétique mais on peut toujours élever suffisamment la température pour observer la région paramagnétique; au contraire quand $w$ est négatif, $w$ est généralement grand en valeur absolue et, quelle que soit la température, on est toujours dans la

(1) En réalité, la formule (3) n'est jamais rigoureusement valable. Avec le modéle proposé, quand $w$ est négatif. le calcul complet montre que $\%$ varie d'une façon compliquée avec la température. A haute température, la courbe est asymptote à la droite représentée par la formule (3) tandis qu'au zéro absolu, on obtient une tangente horizontale ( $\chi$ constant). En gros, on peut dire que la température $T=-\theta$ sépare la courbe en deux portions suivant l'écart qui la sépare de l'une ou de l'autre de ces deux droites. 
région où la formule 3 n'est pas valable, d'où la nécessité d'une étude spéciale de cette région qui sera pour les corps

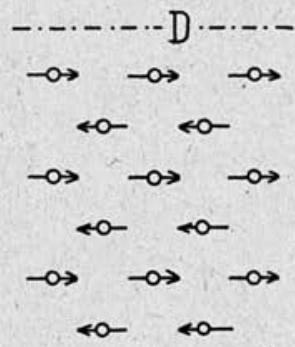

Fig. 8.

à champ moléculaire négatif la réplique de la région ferromagnétique des corps à champ moléculaire positif.

Au zéro absolu, chaque atome se dispose antiparallèlement à ses voisins, de manière à réaliser un assemblage d'énergie

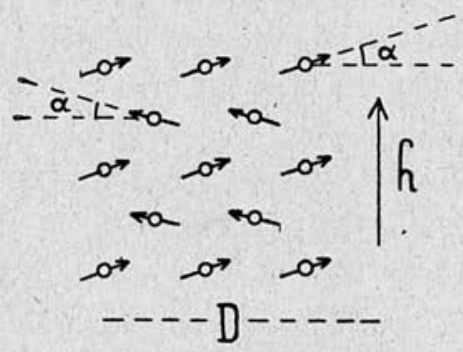

Fig. 9.

potentielle minimum comme celui qui est représenté sur la figure 8. Les moments sont tous parallèles à une même direction $\mathrm{D}$, mais ils sont dirigés dans des sens différents au lieu d'être tous de même sens comme dans les ferromagnétiques. Un champ magnétique $h$, perpendiculaire à la direction D, va déformer cet assemblage et l'aimanter. Tous les 
atomes vont tourner d'un même angle $\alpha$, petit (fig. 9). Soit $2 p$ le nombre des voisins, $\mathrm{N}$ le nombre des atomes, l'énergie totale, somme de l'énergie de déformation et de l'énergie magnétique, est égale à :

$$
\mathrm{W}=+\rho \mathrm{N} \omega\left(1-2 \alpha^{2}\right)-\mu \mathrm{N} / \alpha
$$

Cette expression est minimum pour :

$$
\alpha=\frac{\mu h}{-4 p w}
$$

d'où une aimantation totale :

$$
J=\frac{N \mu^{2} h}{-4 p w} .
$$

C'est l'expression d'un paramagnétisme indépendant de la température.

§18. Rôle des actions cristallines. - D'après les considérations précédentes, si la direction D n'est pas initialement perpendiculaire à la direction du champ magnétique extérieur $h$, les porteurs doivent tourner pour réaliser cette disposition dont l'énergie potentielle est minimum. A cause de la rotation d'ensemble des porteurs il est peu probable que la direction D puisse tourner sans qu'aucune variation d'énergie ne se produise de ce fait. En effet. comme dans les ferromagnétiques, attribuons le moment au spin de l'électron. Ces électrons appartiennent à des orbites bloquées dont les orientations sont fixes par rapport au réseau cristallin. Un faible couplage spin-orbite va suffire à orienter les moments dans une direction privilégiée par rapport aux axes cristallins. C'est ainsi qu'on peut interprèter, d'après Powell (i5), les propriétés des mono-cristaux de nickel et de cobalt.

Pour introduire ces actions, d'une manière aussi simple que possible, je considérerai la substance comme un ensemble de domaines élémentaires possédant chacun une seule 
direction privilégiée D; j'écris l'énergie de couplage entre le moment magnétique et le réseau cristallin sous la forme $w^{\prime \prime} \operatorname{Cos} 2 \theta$ en désignant par $\theta$ l'angle du moment avec la direction privilégiée. $w^{\prime \prime}$ sera beaucoup plus faible que $w$, comme c'est le cas pour les substances ferromagnétiques.

Soit $\beta$ l'angle aigu du champ magnétique extérieur avec la direction privilégiée D. Dans l'état d'équilibre, la moitié des moments (tig. Io) fera avec $\mathrm{H}$ l'angle $\downarrow+\alpha$ et l'autre

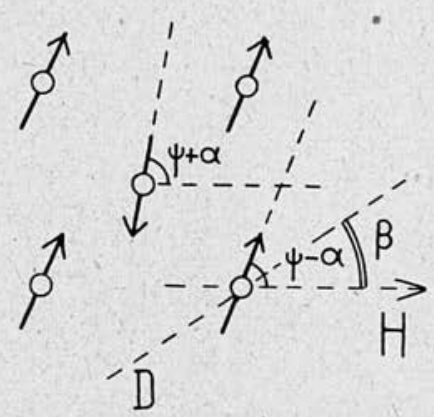

Fig. 10.

moitié l'angle $-(y-\alpha)$. Comme l'énergie magnétique est toujours très faible devant l'énergie d'interaction, l'angle $\alpha$ sera très petit et, par atome, l'énergie de couplage entre le moment et le réseau sera $w^{\prime \prime} \cos 2(\psi-\beta)$, et comme la composante du champ magnétique perpendiculaire à la direction moyenne des moments est $\mathrm{H} \sin \psi$, l'angle de déformation est : $\alpha=\frac{\mu \mathrm{H} \sin \psi}{-4 p w}$ et l'énergie magnétique $-\alpha \mu \mathrm{H} \sin \psi$ l'énergie totale est :

$$
\mathrm{W}=-w^{\prime \prime} \cos 2(\psi-\beta)-\frac{\mu^{2} \mathrm{H}^{2} \sin ^{2} \psi}{-4 p w}
$$

Posons :

$$
\lambda=\frac{\mu^{2} \mathrm{H}^{2}}{8 p w w^{\prime \prime}} \quad \operatorname{tg} 2 \varphi=\frac{\sin 2 \beta}{\cos 2 \beta-\lambda}
$$


On prendra $0<2 \varphi<\frac{\pi}{2}$ quand le second membre de ${ } 7$ est positif et $\frac{\pi}{2}<2 \varphi<\pi$ quand le second membre est négatif. Dans ces conditions, l'énergie est minimum pour $\psi=\varphi$ et l'aimantation relative à $\mathrm{N}$ atomes s'écrit :

$$
\jmath=\frac{N \mu^{2} \mathrm{H}}{-4 p w} \sin ^{2} \varphi .
$$

§19. Discussion de la formule 18. - Cette formule montreque la susceptibilité est une fonction du champ magnétique $\mathbf{H}$ et de l'angle $\beta$. Soit $\mathrm{H}_{0}$ un champ critique défini par l'équation :

$$
\mathrm{H}_{0}^{2}=\frac{-8 p w w^{\prime \prime}}{\mu^{2}}
$$

et posons :

$$
\chi_{m}=\frac{N \mu^{2}}{-4 p w}
$$

La figure I r représente les variations de $\frac{\chi}{\chi_{m}}$ en fonction de $\frac{\mathrm{H}}{\mathrm{H}_{0}}$, pour différentes valeurs de $\beta$ espacées de $10^{\circ}$ en $10^{\circ}$.

Lorsque la direction privilégiée est perpendiculaire au champ magnétique extérieur, la susceptibilité est indépendante du champ et égale à $\chi_{m}$. Au contraire, lorsque la direction d'alignement $D$ est parallèle au champ extériẹur $(\beta=0)$, la susceptibilité est nulle pour les champs inférieurs au champ critique $\mathrm{H}_{0}$ et égale à $\chi_{m}$ pour les champs supérieurs, avec une discontinuité pour $\mathrm{H}=\mathrm{H}_{0}$. Pour les directions intermédiaires, la variation est plus compliquée.

Il est intéressant de remarquer que la susceptibilité reste pratiquement indépendante du champ, soit dans le cas où le champ extérieur est supérieur au double du champ critique, soit dans le cas où il est inférieur au cinquième du champ critique, cela quelle que soit l'orientation de la direction privilégiée. 
PROPRiÉTÉS MAGNÉTIQUES DE L'ÉTAT MÉTALLiQUe 26 I

Pour une substance à cristallisation confuse, deux cas extrêmes sont donc à envisager :

a) Le champ extérieur est très petit. Dans les différents domaines élémentaires, les directions privilégiées sont répar-

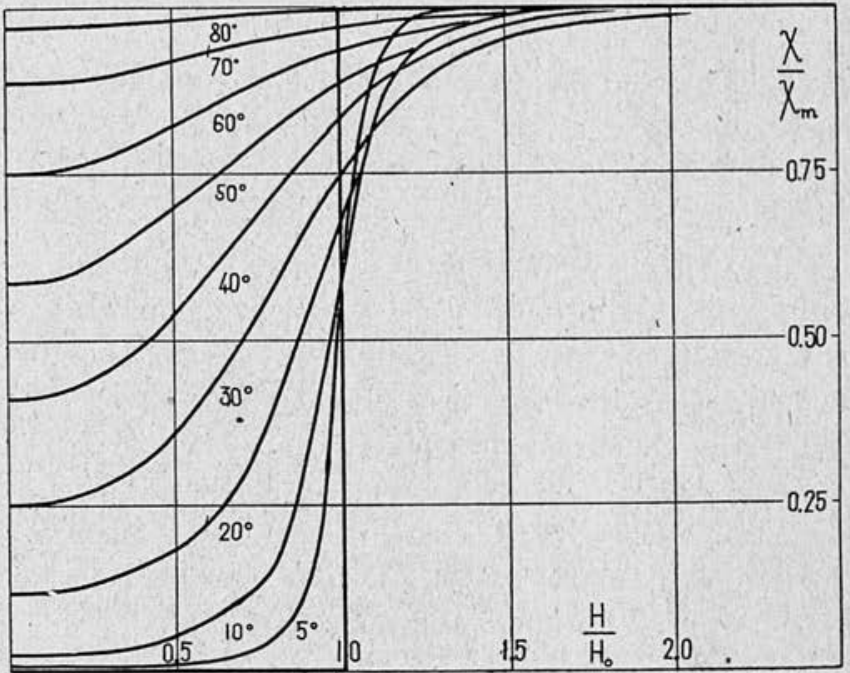

Fig. Ix. - Susceptibilité en fonction du champ, pour différentes orientations de la direction privilégiée.

ties au hasard. Pour chaque direction, la susceptibilité est donnée par la formule 18 dans laquelle $\varphi=\beta$ puisque $\lambda=0$. Pour avoir la susceptibilité moyenne, il faut prendre la valeur moyenne de $\sin ^{2} \beta$. soit $\frac{2}{3}$, et la susceptibilité de l'ensemble s'écrit :

$$
\chi_{m}=\frac{N \mu^{2}}{-6 p w} .
$$

b) Le champ extérieur est très grand. Dans ce cas l'orientation initiale de D n'intervient pas et la susceptibilité moyenne est égale à la susceptibilité maximum.

Les raisonnements précédents sont valables au zéro absolu. 
Pour des températures petites devant $-\theta$, les conclusions précédentes ne sont pas modifiées. A haute température au contraire, dans la région où la loi de Weiss est valable, l'effet du champ cristallin est négligeable, absolument comme dans les ferromagnétiques.

§20. Calcul de l'énergie d'interaction des métaux à paramagnétisme constant. - C'est au mécanisme décrit dans les $\$ \S 17$ et suivants que j'attribue le paramagnétisme indépendant de la température de la plupart des métaux. Ils possèdent ainsi essentiellement un moment atomique et un champ moléculaire négatif. En fonction du moment et de l'énergie d'interaction, la susceptibilité à bảsse température est donnée par les formules 19 ou 20 , suivant les valeurs relatives du champ magnétique extérieur et du champ critique $\mathrm{H}_{0}$. Pour des raisons qui seront développées dans le $\$ 30$, il est probable que, dans la majorité des cas, le champ critique est supérieur aux champs utilisés dans les mesures de susceptibilité ; il faut donc utiliser la formule :

$$
\chi=\frac{N \mu^{2}}{-6 p w}
$$

Si le moment de l'atome est de $q$ magnétons de Bohr, l'énergie d'interaction est égale à $q^{2} w$ et :

$$
\chi=\frac{N q^{2} \mu^{2}}{-6 p q^{2} w}=\frac{N \mu^{2}}{-6 p w} .
$$

La susceptibilité à basse température est indépendante du nombre des électrons couplés parallèlement sur chaque atome. Elle fournit simplement la valeur $w$ de l'énergie d'interaction entre deux électrons placés sur deux atomes voisins.

Il est délicat de déterminer le nombre $2 p$ des voisins efficaces qui interviennent dans la formule 20. 
$\S 21$. Nombre des voisins efficaces. - Deux cas simples peuvent être traités.

a) Réseau du cube centré. - Ce réseau peut être obtenu en entrelaçant deux réseaux cubiques simples identiques : un atome quelconque de l'un des réseaux possède 8 voisins qui appartiennent tous à l'autre réseau. Il est très plausible de supposer qu'au zéro absolu tous les atomes de l'un des réseaux sont dirigés parallèlement à la direction privilégiée et tous les atomes de l'autre réseau en sens inverse. C'est d'ailleurs la disposition qui réalise le minimum d'énergie potentielle. Dans ces conditions le nombre des voisins est égal à 8 et :

$$
\chi=\frac{N \mu^{2}}{-24 w} .
$$

b) Réseau du cube à faces centrées. - Le problème est plus délicat. L'une des dispositions qui parait réaliser le minimum d'énergie polentielle est la suivante : divisons les atomes par plans successifs perpendiculaires à l'un des axes quaternaires; dirigeons les moments, contenus dans l'un des plans parallèlement à la direction privilégiée et les moments contenus dans le plan suivant antiparallèlement et ainsi de suite. Un atome quelconque possède ainsi, dans le même plan, 4 voisins dirigés dans le même sens que lui, et dans les deux plans voisins, 8 voisins dirigés en sens inverse. Dans la petite déformation qui se produit sous l'action du champ magnétique, l'énergie potentielle entre voisins dirigés dans le même sens ne varie pas puisque les moments restent parallèles; tout se passe donc comme si ces voisins n'existaient pas et il faut faire intervenir seulement les 8 voisins antiparallèles, comme dans le réseau du cube centré. La formule 2 I servira aussi à calculer l'énergie.

Comme les métaux qui ne cristallisent pas dans les deux systèmes précédents cristallisent dans des réseaux qui ne s'en écartent néanmoins que fort peu, j'utiliserai dans tous les cas la formule $2 \mathrm{r}$. 
§ 22. Corrections de diamagnétisme. - Le paramagnétisme constant métallique est généralement assez faible, comparable en valeur absolue au diamagnétisme atomique des ions, d'où la nécessité d'une correction relative au diamagnétisme sous-jacent de l'atome.

Les formules semi-empiriques de Slater (16) permettent de calculer le diamagnétisme d'un atome de configuration électronique donnée : voici quelques chiffres relatifs aux éléments de transition (tableau 6).

Tableau 6

\begin{tabular}{|c|c|c|c|c|c|c||}
\hline $\begin{array}{c}\text { Confi- } \\
\text { guration }\end{array}$ & Métal & $-\chi_{\mathrm{A}} \times 10^{6}$ & Métal & $-\chi_{\mathrm{A}} \times 10^{6}$ & Métal & $-\chi_{\mathrm{A}} \times 10^{6}$ \\
\hline & & & & & \\
\hline$d^{8} s^{2}$ & $\mathrm{Ni}$ & 43 & $\mathrm{Pd}$ & 72 & $\mathrm{Pt}$ & $9^{3}$ \\
$d^{7} s^{2}$ & $\mathrm{Co}$ & 46 & $\mathrm{Rh}$ & 77 & $\mathrm{Ir}$ & 99 \\
$d^{6} s^{2}$ & $\mathrm{Fe}$ & 50 & $\mathrm{Ru}$ & 83 & $\mathrm{Os}$ & $\mathrm{I06}$ \\
$d^{5} s^{2}$ & $\mathrm{Mn}$ & 53 & & & & \\
$d^{4} s^{2}$ & $\mathrm{Cr}$ & 57 & & & & \\
$d^{3} s^{2}$ & $\mathrm{~V}$ & $6 \mathrm{I}$ & & & & \\
$d^{2} s^{2}$ & $\mathrm{Ti}$ & 65 & & & & \\
\hline
\end{tabular}

Ces données sont relatives à des atomes neutres libres. A l'état métallique, la part contributive des électrons $s$, en particulier, doit être modifiée fortement. Le seul métal paramagnétique où le diamagnétisme soit connu expérimentalement avec précision est le palladium, les expériences de Kopp donnent $\chi_{\mathrm{A}}=-58.10^{-6}$. La valeur donnée pour le platine est beaucoup moins sûre. La valeur calculée pour le palladium étant $-72 \cdot 10^{-6}$, il faut la multiplier par $0,8 \mathrm{r}$ pour obtenir la valeur expérimentale. J'ai estimé obtenir l'ordre de grandeur du diamagnétisme atomique en multipliant les valeurs du tableau 6 par le même coefficient de réduction que celui du palladium ; c'est ainsi que les nombres du tableau 7 ont été calculés. 


\section{TABLEAU 7}

\begin{tabular}{||c|r|c|c|c|c|c|c|c||}
\hline Métal & $\mathrm{Mn}$ & $\mathrm{Cr}$ & $\mathrm{V}$ & $\mathrm{Ti}$ & $\mathrm{Rh}$ & $\mathrm{Ru}$ & $\mathrm{Ir}$ & $\mathrm{Os}$ \\
$-\chi_{\mathrm{\Lambda}} \times 10^{\circ}$ & 43 & 46 & 49 & 52 & 62 & 67 & 80 & 85 \\
\hline
\end{tabular}

$\S 23$. Valeurs numériques de $w$ pour quelques métaux à paramagnétisme constant. - La deuxième colonne du tableau 8 donne la susceptibilité atomique de quelques métaux. Les valeurs corrigées du diamagnétisme d'après les chiffres du tableau 7 sont portées dans la quatrième colonne. Enfin dans la dernière colonne j'ai calculé $\frac{w}{N \mu^{2}}$ d'après la formule $2 \mathrm{I}$.

Pour l'iridium et l'osmium la correction de diamagnétisme est si grande que les valeurs de $w$ correspondantes sont assez incertaines. Pour le manganèse, j'ai choisi la valeur de la susceptibilité de $\mathrm{Mn}_{\gamma}$, car le réseau cristallin correspondant est tétragonal avec une disposition simple et bien connue des atomes voisins ce qui n'est pas le cas de la phase $\alpha$ où il faut distinguer quatre sortes d'atomes entourés différemment.

\section{Tableau 8}

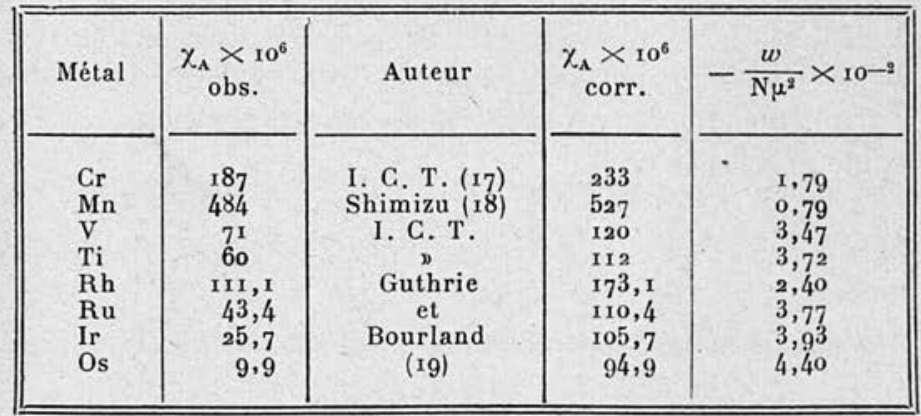

Ann. de Phys., $\mathrm{I}_{1} \mathrm{e}$ série, t. 5 (Février 1936 ). 
§ 24. Paramagnétisme superposé des alliages de nickel. - J'ai attribué ce paramagnétisme superposé à la formation de liaisons antiparallèles entre le moment du nickel et celui du métal étranger introduit. L'énergie d'interaction correspondante peut se calculer connaissant $a$; en effet, soit une solution assez étendue pour que tout atome $X$ du métal étranger ne possède comme voisins que des atomes de nickel, le paramagnétisme constant superposé de la solution solide est dû à des assemblages distincts, séparés les uns des autres, constitués de la façon suivante : un atome $\mathrm{X}$ au centre, avec $q$ électrons dirigés parallèlement à une certaine direction, tout autour $q$ électrons appartenant à des atomes de nickel dirigés antiparallèlement. Soit $-w \cos \alpha$, l'énergie d'interaction de deux électrons; à basse température, la susceptibilité de cet assemblage est ;

$$
\chi=\frac{2 q^{2} \mu^{2}}{-3 q^{2} w}
$$

(c'est la formule 20 bis dans laquelle $2 p=\mathrm{r}$ et $\mathrm{N}=2$ ).

Soit $\tau$ le titre atomique du métal $X, \mathrm{~N}$ le nombre d'Avogadro, la susceptibilité atomique s'écrit :

$$
\chi_{\mathrm{A}}=\frac{2 \tau N \mu^{2}}{-3 w} .
$$

Cette formule n'est applicable qu'aux faibles concentrations, puisque, comme je l'ai signalé (§ 9), le nombre de liaisons par atome augmente avec la concentration à cause des interactions entre assemblages différents qui finissent par bloquer tous les atomes en un réseau complet. La formule générale s'écrit :

$$
\chi_{\mathrm{A}}=\frac{{ }^{2 \tau N \mu^{2}}}{3 w(\mathrm{I}+\mathrm{II} \tau)} .
$$

J'ai calculé la valeur moyenne $m$ de $\frac{\chi_{A}(x+11 \tau)}{\tau}$ pour les différentes séries d'alliages étudiés et les résultats sont don- 
nés dans le tableau 9 ainsi que les valeurs de $\frac{w}{N \mu^{2}}$ correspondantes.

\section{TABLEAU 9}

\begin{tabular}{|c|c|c|}
\hline \hline Liaison & $m$ & $-\frac{w}{N \mu^{2}} \times 10^{-2}$ \\
\hline Ni-Al & 177 & 3,76 \\
Ni-Mo & 404 & 1,67 \\
Ni-W & 401 & 1,66 \\
Ni-V & 390 & $1.6 \mathrm{r}$ \\
Ni-Ti & 172 & 3,87 \\
Ni-Cr & 410 & 1,62 \\
\hline \hline
\end{tabular}

$\S 25$. Energie d'interaction et distance des couches magnétiques. - J'ai rassemblé dans le tableau 10 les valeurs de $w$ calculées dans les paragraphes précédents.

On remarque que dans la première série des éléments de transition, le champ moléculaire croìt régulièrement depuis le titane jusqu'au nickel; de même dans les séries $\mathrm{Ru}, \mathrm{Rh}$, $\mathrm{Pt}$ et $\mathrm{Os}, \mathrm{Ir}, \mathrm{Pt}$.

Slater (16) a rapproché les propriétés ferromagnétiques du fer, du nickel et du cobalt de la petitesse du diamètre de leur couche magnétique - couche $3 d$ - vis-à-vis de la distance de deux atomes voisins dans le cristal. Soit $\delta$ le diamètre de cette couche, $d$ la distance de deux voisins : Stoner (8) a précisé que les énergies d'interaction variaient régulièrement avec le rapport $\frac{d}{\delta}$. J'ai précisé cette remarque (20) en l'étendant aux liaisons Cr-Cr, Mn-Mn, Ni-Co, Ni-Fe, Co-Fe. Mais j'ai remarqué depuis que les énergies d'interaction pouvaient tout aussi bien être considérées comme fonction de l'unique variable $d-\delta$, plus petite distance entre les deux couches magnétiques. 
TABleau 10

\begin{tabular}{|c|c|c|c|c|}
\hline Liaison & $\frac{w}{N \mu^{2}} \times 10^{-2}$ & $d$ & $\delta$ & $d-\hat{\delta}$ \\
\hline $\mathrm{Ti} \cdot \mathrm{Ti}$ & $-3,7^{2}$ & 2,92 & 2,46 & 0,46 \\
\hline V-V & $-3,47$ & 2,63 & 2,09 & 0,54 \\
\hline $\mathrm{Cr}-\mathrm{Cr}$ & $-\quad \mathrm{I}, 79$ & $=\quad 2,49$ & 1,83 & 0,67 \\
\hline $\mathrm{Mn}-\mathrm{Mn}$ & $-0,79$ & 2,58 & 1,61 & 0,97 \\
\hline $\mathrm{Fe}-\mathrm{Fe}$ & - o,33 & 2,57 & 1,44 & $\mathrm{I}, \mathrm{13}$ \\
\hline Co. Co & $+\quad 1,02$ & 2,49 & 1,30 & 1,19 \\
\hline $\mathrm{Ni}-\mathrm{Ni}$ & $+1,67$ & 2,49 & 1,19 & 1,30 \\
\hline$R u-R u$ & $-3,77$ & 2,69 & 2,19 & 0,50 \\
\hline Rh-Rh & $\begin{array}{l}2,40 \\
-\end{array}$ & 2,69 & $\mathrm{I}, \mathrm{l}, 8$ & $0,7^{1}$ \\
\hline Pd-Pd & $-0,59$ & 2.74 & 1,82 & $0,9^{2}$ \\
\hline Os-os & $-4,40$ & 2,71 & 2,54 & 0,17 \\
\hline $\mathrm{Ir} \cdot \mathrm{Ir}$ & $-3,9^{3}$ & $2,7^{6}$ & 2,32 & 0,44 \\
\hline $\mathrm{Pt} \cdot \mathrm{Pt}$ & $-2,88$ & 2,76 & 2,12 & 0,64 \\
\hline $\mathrm{Ni}$-Co & $+\mathbf{1}, 58$ & 2,49 & 1,25 & I, 24 \\
\hline $\mathrm{Co}-\mathrm{Fe}$ & $+0,60$ & 2,49 & $\mathrm{I}, 37$ & 1,12 \\
\hline $\mathrm{Ni} \cdot \mathrm{Fe}$ & $+1,13$ & 2,49 & 1,30 & 1,19 \\
\hline $\mathrm{Ni}-\mathrm{Ti}$ & $-3,87$ & 2,49 & 1,82 & 0,67 \\
\hline $\mathrm{Ni}-\mathrm{V}$ & $-1,6 i$ & 2,49 & 1,64 & 0,85 \\
\hline
\end{tabular}

Les formules de Slater permettent de calculer les diamètres des différentes couches électroniques d'un atome de configuration donnée. J'ai calculé les diamètres de la couche $d$, couche magnétique, en admettant les configurations données dans le tableau 6 . Ce sont les $\delta$ donnés dans la quatrième colonne du tableau 10, exprimés en angströms. Dans la troisième colonne, je donne les distavces $d$, en angströms, entre deux atomes voisins dans le réseau cristallin. Les différences $d$ - $\delta$ sont portées dans la dernière colonne. Pour les liaisons mixtes, j’ai pris la moyenne des diamètres des couches magnétiques des deux atomes, comme valeur de $\delta$.

La figure 12 représente les valeurs de l'énergie d'interaction en fonction de $d-\delta$. La régularité des résultals est aussi grande qu'on pouvait l'espérer étant donné l'incertitude qui s'attache au calcul du rayon de la couche magnétique.

Cette régularité ne doit cependant pas faire illusion, car, dans la différence $d-\delta$, si $\delta$ varie dans le rapport de $\mathrm{I}$ à $\mathbf{2}$, 
PROPRIÉtÉS MagNétiques de L'État MÉTALLique $\quad 269$

$d$ ne varie que dans le rapport de $\mathrm{I}$ à $\mathrm{I}, \mathrm{I}$. En fait, si on représente $w$ en fonction de $\frac{d}{\delta}$ la courbe obtenue a exactement la même allure que la précédente. La régularité observée móntre surtout que le champ moléculaire dépend du diamètre des couches magnétiques.

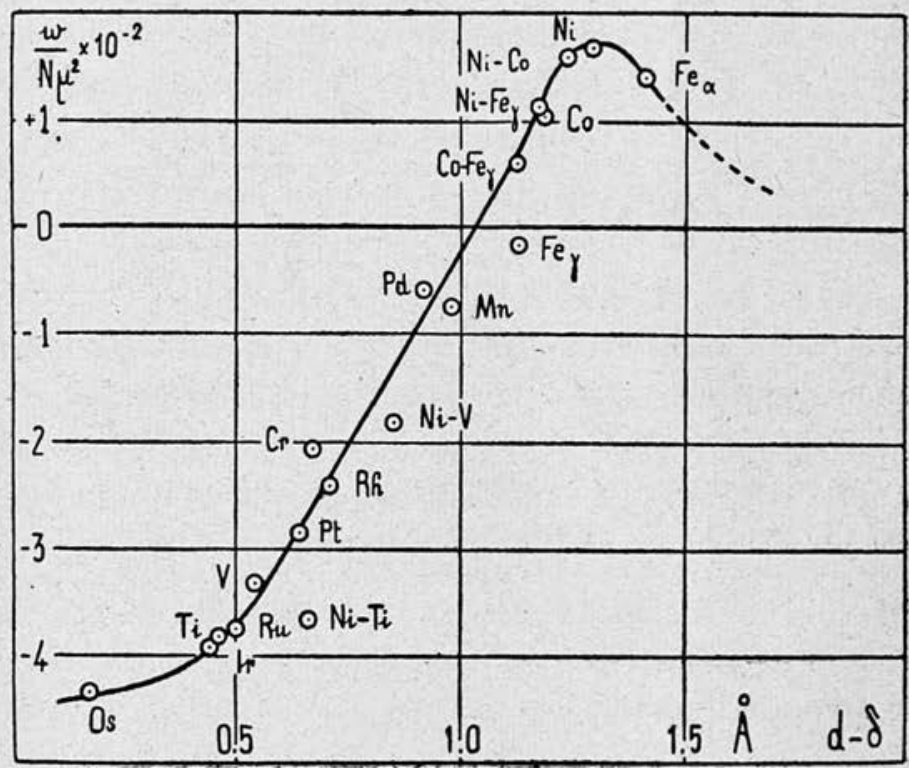

Fig. 12. - Le champ moléculaire en fonction de la distance des couches magnétiques.

Mais les arguments suivants montrent que $w$ varie également avec la distance :

a) Normalement, dans le manganèse, la distance entre atomes voisins est de $2,58 \AA$; le champ moléculaire est négatif. Si on fait une solution étendue de manganèse dans du cuivre où $d=2,55 \AA$, deux atomes de manganèse accidentellement voisins posséderont un champ moléculaire négatif; au contraire une solution de manganèse dans l'argent 
$(d=2,88 \AA)$ donnera $d-\delta=1,27 \AA$ correspondant à une interaction positive. On constate en effet (21) qu'aux faibles dilutions le champ moléculaire des solutions solides $\mathrm{Mn}-\mathrm{Cu}$ est négatif, tandis qu'il est positif pour les solutions Mn-Ag.

b) Sadron a trouvé (I) que dans les $\mathrm{Mn}-\mathrm{Ni}$, le manganèse est orienté parallèlement par le nickel. En effet, la distance entre la couche magnétique d'un atome de manganèse et la couche magnétique d'un des atomes de nickel voisins est 2,49-1,40 = 1,09 $\AA$ correspondant à une interaction positive; au contraire dans les solutions de manganèse dans le cobalt: $d-\delta=\mathrm{r}, 03 \AA$ correspondant à une interaction négative ; effectivement Mn est orienté antiparallèlement par Co ( $\mathrm{I}$ ).

c) Dans le fer $\alpha$ (fer à la température ordinaire), un atome de fer possède 8 voisins à une distance de 2,48 A et 6 voisins à une distance de $2,86 \AA$. Les $d-\delta$ correspondants sont respectivement ${ }_{1}, 04 \AA$ et $1,42 \AA$. La première différence correspond d'après la figure 12 à une interaction sensiblement nulle. Le champ moléculaire positif du fer doit provenir des 6 voisins les plus éloignés. La valeur correspondante de $\frac{w}{N \mu^{2}}$ est $1,38.10^{2}$, qui n'est pas en désaccord avec la courbe de la figure 12. Elle semble montrer que, pour des distances entre couches magnétiques supérieures à 1,40 A, l’énergie d'interaction décroît avec la distance, résultat très naturel. Il est très remarquable que M. Forrer ( 23 ), par des considérations essentiellement différentes, ait déjà montré que le champ moléculaire du fer est produit par les voisins situés à la distance de $2,86 \AA$.

d) Pour le fer dissous dans l'or $(d=2,88 \AA)$, deux atomes de fer accidentellement voisins donnent $d-\delta=\mathrm{r}, 44 \AA$, donc une interaction positive. Les solutions solides $\mathrm{Fe}-\mathrm{Au}$ ont en effet un point de Curie positif (22).

$e)$ De la figure 12 on déduit que la dilatation thermique 
augmente le champ moléculaire de tous les métaux sauf le fer $\alpha$. Quand le champ moléculaire est négatif, sa valeur absolue diminue avec la température, la susceptibilité doit augmenter. C'est peut-être de cette façon qu'il faut expliquer la légère augmentation avec la température de la susceptibilité des métaux tels que V, Ti, Os, Ir, Ru, Rh.

Malheureusement, la grandeur exacte de cet effet ne peut être calculée car la température, non seulement augmente la constante du réseau, mais accroit l'amplitude des oscillations des atomes autour de leur position d'équilibre, d'où modification de l'énergie moyenne d'interaction puisqu'elle n'est pas une fonction linéaire de la distance. A des températures de $1000^{\circ} \mathrm{K}$, l'amplitude de ces oscillations atteint quelques dixièmes d'angstrœm ; la modification de l'énergie d'interaction qui en résulte peut être importante. Dans les corps à champ moléculaire positif, les variations du champ moléculaire modifient la constante de Curie; il faut en tenir compte dans l'interprétation de la valeur de la constante de Curie.

§ 26. Remarques. - Les valeurs connues de l'énergie d'interaction sont assez sûres lorsque la distance des couches magnétiques est comprise entre $0,1 \AA$ et $1,5 \AA$. Au delà de ces limites la courbe est difficile à préciser. Quand $d-\delta$ augmente, $w$ doit tendre vers zéro après avoir pris peut-être des valeurs faiblement négatives.

Pour des valeurs de $d-\delta$ inférieures à $\mathrm{o}, \mathrm{r} \AA$, on pourrait utiliser le molybdène $(d-\delta=-0,04 \AA)$ mais le diamètre de sa couche magnétique est si grand $(2,77 \AA)$ que les actions entre atomes qui ne sont pas proches voisins ne sont plus négligeables. Les résultats obtenus ne pourraient être qu'illusoires.

Retenons surtout de ces résultats que le domaine du ferromagnétisme est assez étroitement limité aux distances entre couches magnétiques comprises entre I, I et I, $5 \AA$. 
$\S 27$. Températures de dégénérescence. - L’inverse $\frac{1}{\chi}$ de la susceptibilité du modèle employé dans les paragraphes i 7 et suivants est une fonction de la température représentée sur la figure 13 . Pour des températures de l'ordre de $-\theta$, existe une région de transition entre le paramagnétisme constant et le paramagnétisme à champ moléculaire de

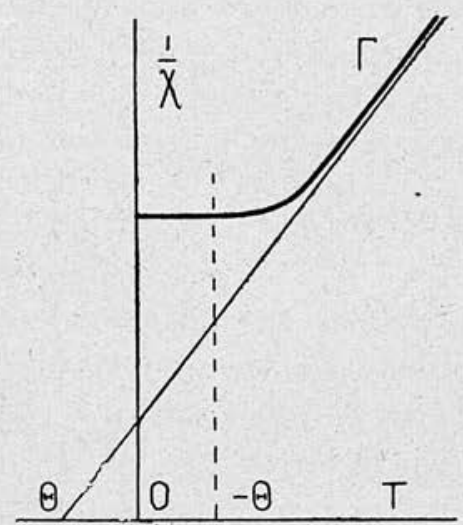

Fig. 13 .

Weiss. Le manganèse et le chrome sont normalement des paramagnétiques constants mais en les diluant dans des métaux comme l'or, l'argent ou le cuivre on diminue assez le champ moléculaire pour que les alliages obéissent à la loi de Weiss et confirment ainsi les hypothèses fondamentales (2I).

Peut-on espérer pour un métal pur élever suffisamment la température pour attẹindre la région où la susceptibilité varie avec la température ? Le point de Curie se calcule d'après la formule 5 mise sous la forme :

$$
\theta=2 p C \frac{w}{N \mu^{2}} .
$$

On peut admettre comme constantes de Curie du manganèse et du chrome, respectivement les valeurs 2,9 et 2,8 , 
déduites de l'étude du métal en solution étendue. On trouve ainsi pour le chrome $-4000^{\circ}$ et le manganèse $-1800^{\circ}$. Pour le chrome, il est sûr que jusqu'aux plus hautes températures atteintes expérimentalement on ne dépasse pas la partie horizontale de la courbe $\Gamma$. En effet la susceptibilité du chrome est indépendante de la température. Pour le manganèse, je suis moins affirmatif, car je ne connais pas exactement la courbe $\Gamma$ mais la tangente au zéro absolu et l'asymptote. La courbe tracée sur la figure 13 représente la susceptibilité d'un groupe de deux atomes seulement aux moments antiparallèles (cf. 9 p. 66) et non pas celle d'un réseau spatial d'atomes qui correspond pourtant mieux à la réalité.

D'ailleurs, au point de vue expérimental, si Honda et Soné (24) attribuent au manganèse un paramagnétisme tout à fait indépendant de la température, au contraire les expériences de Shimizu ( 18$)$, qui sont plus récentes et qui paraissent plus sûres, montrent que chacune des trois variétés de manganèse possède un paramagnétisme qui décroit- avec la température.

§ 28. Températures de dégénérescence de quelques liaisons mixtes. - L'ordre de grandeur des températures de dégénérescence des liaisons mixtes se calcule par la formule ${ }_{2} 3$ avec $2 p=\mathrm{r}$. Il s'agit bien entendu des assemblages antiparallèles, séparés les uns des autres, qui se forment en solution très étendue et non pas d'un réseau antiparallèle complet dont la température de dégénérescence dépend du nombre des liaișons. La constante de Curie qui figure dans la formule 23 est celle du métal allié au nickel.

Etudions la liaison $\mathrm{Ni}-\mathrm{Cr}$; la distance calculée entre couches magnétiques est $2,49-1,50=0,99 \AA$ qui correspond d'après la courbe moyenne de la figure $\mathbf{2} 2$ dे :

$$
-\frac{w}{N \mu^{2}}=0,4 \times 10^{2}
$$


d'où on déduit : $\Theta=-2,8 \times 40=-1$ I $2^{\circ}$. Dès la température ordinaire, cette liaison possédera un paramagnétisme variable, ce qu'on peut exprimer d'une íaçon plus suggestive en disant qu'elle subit un commencement de dissociation. Le nombre des atomes de nickel qui, dans l'alliage, voient leurs propriétés magnétiques annulées par des atomes de chrome est donc moins grand que s'il n'y avait pas de dissociation. L'abaissement $-\Delta \mathrm{C}$ de la constante de Curie du nickel, produit par le chrome, doit ainsi être inférieur à celui que produisent ses homologues, tungstène et molybdène. En effet, pour $\mathrm{Cr}$ on a : $-\Delta \mathrm{C}=\mathrm{r}, 6$ et pour $\mathrm{W}$ : $-\Delta \mathrm{C}=3$, og pour Mo: $-\mathrm{JC}=3$, o4. Corrélativement le paramagnétisme constant superposé, observé expérimentalement, doit être rapporté à un nombre de liaisons moindre que celui gu'on prévoit sans dissociation. Le calcul donne donc une valeur trop forte de $-\frac{w}{N \mu^{2}}$. J'avais en effet trouvé : $\mathbf{1}, 62 \times \mathrm{IO}^{2}$, valeur trop grande puisque égale à celle de la liaison $\mathrm{Ni}-\mathrm{V}$.

Pour les autres liaisons mixtes, les valeurs de $\Theta$ sont beaucoup plus grandes. Par exemple, pour le titane. on trouve $-\Theta=2,98 \times 387=1.150^{\circ}$, en prenant comme constante de Curie celle qui correspond à 4 électrons couplés rigidement, par atome. La dissociation correspondante est négligeable.

\section{§ 29. Dégénérescence à basse température des métaux à} champ moléculaire négatif. - Les métaux à champ moléculaire négatif, platine et palladium, que nous savons obéir dans les circonstances normales à la loi de Weiss, doivent se transformer progressivement en paramagnétiques constants, lorsque la température décroît vers le zéro absolu. Le palladium ne convient guère pour vérifier les prévisions de la théorie : son point de Curie n'estqu'à $-200^{\circ} \mathrm{K}$ de sorte que la région d'anomalie est bien au-dessous de la température 
ordinaire. Au contraire, le point de Curie du platine étant à $-1.100^{\circ} \mathrm{K}$, cette région d'anomalie doit être facilement accessible. En effet, les expériences de Foëx montrent que dans un champ de 6.900 gauss, à $100^{\circ} \mathrm{K}$, l'inverse $\frac{\mathrm{I}}{\chi}$ de la susceptibilité varie près de trois fois moins vite avec la température qu'à $500^{\circ} \mathrm{K}$.

$\$ 30$. Effet du couplage spin-orbite. - Mais le platine présente des anomalies encore plus curieuses. Les très nombreuses et très minutieuses expériences de $M$. Foëx et Mlle Collet (25) ont montré l'existence de plusieurs variétés magnétiques de platine dépendant du champ magnétique. Cette multiplicité d'états se manifeste à basse température (entre $100^{\circ} \mathrm{K}$ et $300^{\circ} \mathrm{K}$ ); à haute température il n'y a qu'une seule variété magnétique (à 8 magnétons) indépendante du champ. En gros, disons qu'à $100^{\circ} \mathrm{K}$ la susceptibilité augmente avec le champ magnétique comme en témoignent les chiffres suivants :

Tableau $1 \mathrm{I}$

\begin{tabular}{|c|c|c|c|}
\hline H en gauss & 6900 & 9300 & 14300 \\
\hline$\chi \times 10^{6}$ & 1,23 & 1,27 & 1,33 \\
\hline \hline
\end{tabular}

Je crois qu'il faut attribuer cette variation au couplage entre les spin et le réseau cristallin dont j’ai précisé le mécanisme dans les paragraphes 18 et suivants.

Dans un champ faible et à très basse température, les directions privilégiées des différents domaines sont distribuées statistiquement suivant toutes les directions de l'espace, la susceptibilité est donnée par la formule:

$$
\chi_{1}=\frac{N \mu^{2}}{24 w} \text {. }
$$


Dans un champ fort, au contraire, les directions d'alignement quittent les directions privilégiées pour venir se placer perpendiculairement au champ magnétique, la susceptibilité de ce nouvel assemblage s'obtient en multipliant la valeur précédente par $\frac{3}{2}$ et on trouve :

$$
\chi_{2}=\frac{N \mu^{2}}{16 w} .
$$

A haute température, quand la régularité de l'assemblage s'est détruite sous l'influence de l'agitation thermique, les énergies de couplage avec le réseau cristallin deviennent négligeables et il n'y a plus de différences entre les susceptibilités dans les champs forts et dans les champs faibles et les deux courbes qui donnent la susceptibilité en fonction de la température dans les deux cas extrêmes possèdent la même asymptote :

$$
\chi=\frac{1}{3} \frac{N \mu^{2}}{k T-2 p \frac{w}{3}} \quad \text { avec } p=6 .
$$

L'allure des deux courbes est donnée par la figure 14 sans que je puisse exactement préciser leur tracé. Lorsque la valeur du champ magnétique est voisine de celle du champ critique $\mathrm{H}_{0}$ les susceptibilités observées doivent être comprises entre ces deux courbes.

En comparant les courbes de la figure 14 et les chiffres du tableau précédent, j'estime que l'ordre de grandeur du champ critique est de 20.000 gauss. En appliquant la formule i 9 on trouve comme énergie de couplage entre les spin et le réseau la valeur :

$$
\mathrm{N} w^{\prime \prime}=\frac{\mathrm{H}_{0}^{2}}{8 p} \cdot \frac{\mathrm{N} \mu^{2}}{w}=0,43 \times 10^{5} \text { ergs. }
$$

Cette valeur est rapportée à $\mathrm{N}=6,06 \times 10^{23}$ liaisons.

Elle est du même ordre de grandeur que l'énergie de couplage spin-orbite du nickel qui est de $3,3 \times 10^{5}$ ergs (voir 
par exemple Stoner, Magnetism and Matter, p. $\left.3{ }_{95}^{5}\right)$. Le mécanisme proposé est donc très vraisemblable. Remarquons que ces énergies de couplage sont d'un ordre de grandeur beaucoup plus petit que les énergies d'interaction; pour le platine, on a e.1 effet : $-\mathrm{N} w=8,9 \times 10^{9}$ ergs; l'énergie d'interaction est plus de 100.000 fois plus grande que

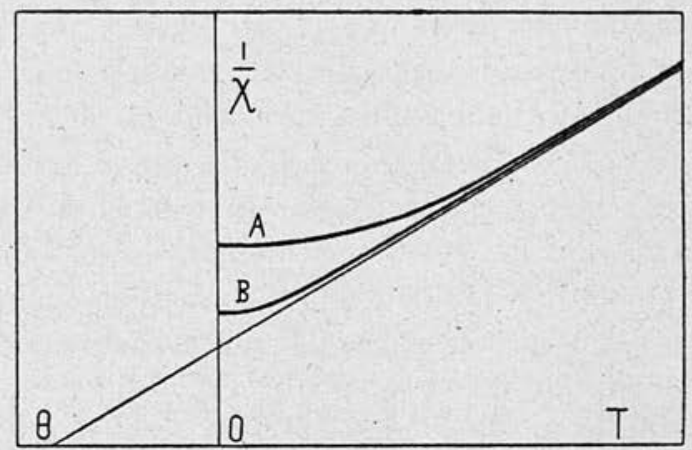

Fig. 14. - A : susceptibilité dans les champs faibles;

B : susceptibilité dans les champs forts.

l'énergie de couplage avec le réseau ce qui montre bien que les deux mécanismes sont entièrement distincts.

Le fer et le cobalt qui sont à la gauche du nickel dans la classification périodique des éléments ont des énergies de couplage spin-orbite près de dix fois plus grandes. Il doit en être de même pour le platine vis-à-vis des métaux qui sont à sa gauche : osmium et iridium. C'est pourquoi j'ai supposé $(\S 20)$ que les champs magnétiques dans lesquels ces métaux ont été étudiés étaient petits vis-à-vis du champ critique et j’ai généralisé pour les autres paramagnétiques constants. La question mériterait une étude expérimentale.

$\$ 31$. - Au terme de cet article, on peut formuler les conclusions générales suivantes.

La valeur de l'aimantation à saturation du nickel indique 
qüil y a, en moyenne, o,6 1 électron par atome responsable des propriétés magnétiques. L'étude des alliages montre que ce sont ces mêmes électrons, ni plus ni moins, qui contribuent au paramagnétisme. Le nombre des porteurs de magnétisme ne change pas au point de Curie.

J'ai tenté de représenter systématiquement les propriétés magnétiques de l'état métallique en attribuant à chaque métal un moment atomique et un champ moléculaire. L'examen des données expérimentales montre que l'énergie d'interaction entre deux atomes ne dépend que de la distance entre leurs couches magnétiques. La légitimité des hypothèses de départ est confirmée par la cohérence entre des résultats obtenus de façons très dissemblables, en particulier l'accord entre les valeurs de l'énergie d'interaction obtenues avec des corps à paramagnétisme constant et des corps obéissant à la loi de Weiss. D'ailleurs l'utilité de ces hypothèses s'affirme par le nombre des faits expérimentaux qui viennent se ranger dans le même système d'interprétation : paramagnétisme de Weiss, paramagnétisme constant, propriétés magnétiques des alliages $\mathrm{Ni}-\mathrm{Co}$, Co.Pt etc..., paramagnétisme superposé des alliages de nickel, résultats de Sadron et de Manders, etc...

Un parallèle entre le ferromagnétisme et le paramagnétisme constant m'a conduit à préciser le rôle des actions cristallines, couplage spin-orbite, et m'a indiqué dans quelle voie il faut rechercher l'explication des propriétés compliquées du platine.

Une théorie aussi primitive et aussi rudimentaire que celle que j'ai développée ici fournit ainsi des résultats assez satisfaisants. Je souhaiterais vivement que tous les problèmes soulevés dans cet article soient traités à nouveau par les méthodes rigoureuses de la mécanique ondulatoire en particulier les variations de l'intégrale d'interaction avec la distance et la dégénérescence à basse température des paramagnétiques à champ moléculaire négatif. 


\section{BIBLIOGRAPHIE}

t. C. Sadron. - Ann. de Physique, 17, 1932, p. 37 I.

2. R. S. Manders. - Ann. de Physique, 5,1936, p. ${ }_{16} 7$.

3. A. Serres - Ann. de Physsique, 17, 1932, p. 5.

4. M. Alder - Thèse, Zurich, r9i6.

5. J. Safranek. - Revue de Métallurgie, fév. 1924.

6. J. Dorman. - Phys. Z/s. d. Sowjet Union, 3, ig33, p. 299.

7. A. Wolf, $-Z$ ts. $f$. Phlys., 70, г93 г, p. 519 .

8. C. Stoner. - Phil. Mag., 15, 1932, p. Io18.

9. L. NÉEL. - Ann. de Physique, 17, r932, p. 5.

10. A. Preuss. - Thèse, Zurich, 1912.

11. M. Peschard. - Thèse, Strasbourg, 1925.

12. W. Kopp. - These, Zurich, 19ig.

13. L. NéEc. - Comm. Soc. Franç. de Phys., Bull. no 355, 1934.

14. O. Веосн, - Thèse, Zurich, I912.

15. F. G. Powell. - Proc. Roy. Soc., 130, r93o, p. 167 ; Proc. Camb. Phil. Soc., 27, ig3ı, p. 56 .

16. J. C. Slater. - Phys. Rev., 36,1930 , p. 57.

17. International Critical Tables, New-York.

18. Y. Sнimizu. - Sc. Rep. Tohóku Imp. Univ., 19, г93o, p. 411.

19. A. N. Guthrie et L. T. Bourland. - Phys. Rev., 37, 1931 , p. 303 .

20. L. NÉEL. - G. R., 198, 1934, p. 1311 .

21. L. NÉEL. - J. de Physique, 3, rg32, p. г6o.

22. J. W. Sнгн. - Phys. Rev., 38, 1931, p. 2051.

23. R. Forrer. - J. de Physique, 4, 1933, p. 109.

24. K. Honda et Soné. - Sc. Rep. Tóhoku Imp. Univ., 2, 1913, p. $\mathbf{r}$.

25. P. Gollet et C. Fö̈x. - C.R., 192, г 13 I, p. 930 ; G. Foëx. J. de Physique, 2, 193 I , p. 353 ; G. Fö̈x. - Les lois expérimentales du paramagnétisme. Mémorial des Sciences Physiques (Paris, Gautier-Villars, 1935). 